\title{
ISOLATED WOLF-RAYET STARS AND O SUPERGIANTS IN THE GALACTIC CENTER REGION IDENTIFIED VIA PASCHEN- $\alpha$ EXCESS
}

\author{
J. C. Mauerhan ${ }^{1}$, A. Cotera ${ }^{2}$, H. Dong ${ }^{3}$, M. R. Morris ${ }^{4}$, Q. D. Wang ${ }^{3}$, S. R. Stolovy ${ }^{1}$, and C. Lang ${ }^{5}$ \\ ${ }^{1}$ Infrared Processing and Analysis Center, California Institute of Technology, Mail Code 220-6, 1200 East California Boulevard, \\ Pasadena, CA 91125, USA; mauerhan@ipac.caltech.edu \\ 2 SETI Institute, 515 North Whisman Road, Mountain View, CA 94043, USA \\ ${ }^{3}$ Department of Astronomy, University of Massachusetts, Amherst, MA 01003, USA \\ ${ }^{4}$ Department of Physics and Astronomy, University of California, Los Angeles, CA 90095, USA \\ 5 Department of Physics and Astronomy, University of Iowa, Iowa City, IA 52245, USA \\ Received 2010 July 27; accepted 2010 September 13; published 2010 November 17
}

\begin{abstract}
We report the discovery of 19 hot, evolved, massive stars near the Galactic center region (GCR). These objects were selected for spectroscopy owing to their detection as strong sources of Paschen- $\alpha(\mathrm{P} \alpha)$ emission-line excess, following a narrowband imaging survey of the central $0.65 \times 0.25(l, b)$ around Sgr A* with the Hubble Space Telescope. Discoveries include six carbon-type (WC) and five nitrogen-type (WN) Wolf-Rayet stars, six O supergiants, and two B supergiants. Two of the $\mathrm{O}$ supergiants have X-ray counterparts having properties consistent with solitary $\mathrm{O}$ stars and colliding-wind binaries. The infrared photometry of 17 stars is consistent with the Galactic center distance, but 2 of them are located in the foreground. Several WC stars exhibit a relatively large infrared excess, which is possibly thermal emission from hot dust. Most of the stars appear scattered throughout the GCR, with no relation to the three known massive young clusters; several others lie near the Arches and Quintuplet clusters and may have originated within one of these systems. The results of this work bring the total sample of Wolf-Rayet (WR) stars in the GCR to 88. All sources of strong P $\alpha$ excess have been identified in the area surveyed with HST, which implies that the sample of WN stars in this region is near completion, and is dominated by late (WNL) types. The current WC sample, although probably not complete, is almost exclusively dominated by late (WCL) types. The observed WR subtype distribution in the GCR is a reflection of the intrinsic rarity of early subtypes (WNE and WCE) in the inner Galaxy, an effect that is driven by metallicity.
\end{abstract}

Key words: Galaxy: center - infrared: stars - stars: emission-line, Be - stars: Wolf-Rayet - supergiants - X-rays: stars

Online-only material: color figure

\section{INTRODUCTION}

The Galactic center region (GCR) has the highest star formation rate density in the Milky Way, more than $\approx 2$ orders of magnitude above the average value of the Galactic disk (Figer et al. 2004). This is considered to be a consequence of the large reservoir of molecular gas that fills the central half-kiloparsec of the Galaxy, where $\approx 10 \%$ of the Galaxy's molecular gas is concentrated. With respect to the physical properties that are typical of molecular gas further out in the Galactic disk, the GCR medium is significantly hotter, more turbulent, and more highly magnetized. It is also subject to mechanical shearing by the strong tidal field, and repeated compression from cloud-cloud collisions, stellar winds, and supernova shock waves (Morris $\&$ Serabyn 1996). It has been suggested that the environmental conditions in the GCR result in an elevated Jeans mass and an enhanced rate of massive-star production.

At least three extraordinary clusters of massive stars have formed within the unique GCR medium during the past several Myr, including the Arches cluster (Nagata et al. 1995) and the Quintuplet cluster (Okuda et al. 1990; Nagata et al. 1990; Glass et al. 1990), both of which lie $\approx 30 \mathrm{pc}$, in projection, from the center. Within the inner few parsecs of the Galaxy lies the Central cluster (Krabbe et al. 1991), which is gravitationally bound to the Galactic black hole, Sgr A* (Schödel et al. 2002; Ghez et al. 2003). All three of the known clusters are massive $\left(M \sim 10^{4} M_{\odot}\right)$ and dense, containing hundreds of $\mathrm{O}$ stars, and dozens of evolved Wolf-Rayet stars (WRs) and OB supergiants (Figer et al. 1999b, 2002; Paumard et al. 2006). The collection of three such extraordinary clusters in the GCR is evidence favoring the hypothesis that the predominant mode of star formation in the region gives rise to dense, massive clusters. However, surveys aimed at identifying massive stars throughout the greater GCR have revealed a population of several dozen massive stars that have no apparent spatial association with the known stellar clusters (Cotera et al. 1999; Homeier et al. 2003; Muno et al. 2006; Mikles et al. 2006; Mauerhan et al. 2007, 2010a, 2010b). The discovery of these "isolated" massive stars has motivated the continued search for additional such cases, with the goal of determining the total extent of the inter-cluster population of massive stars and their physical relation to, or independence from, starburst clusters like the Arches and Quintuplet. The demography and kinematics of these isolated stars will yield valuable insight into the mode of massive star formation in the GCR and the dynamical evolution of massive stellar clusters within galactic nuclei.

The identification of isolated massive stars in the GCR is a difficult task. Heavy extinction limits observations of the Galactic center starlight to the infrared part of the electromagnetic spectrum. Infrared measurements sample the Rayleigh-Jeans tail of stellar spectral energy distributions where there is a degeneracy of stellar color with effective temperature. This renders early-type and late-type stars photometrically indistinguishable. 
Table 1

Basic Data for New Emission-line Stars

\begin{tabular}{|c|c|c|c|c|c|c|c|c|}
\hline No. & Name & $\begin{array}{l}\text { R.A. } \\
\text { (deg, }\end{array}$ & Decl. & $\begin{array}{c}J \\
(\mathrm{mag})\end{array}$ & $\begin{array}{c}H \\
(\mathrm{mag})\end{array}$ & $\begin{array}{c}K_{S} \\
(\mathrm{mag})\end{array}$ & $\begin{array}{c}\text { F190N } \\
(\mathrm{mJy})\end{array}$ & F187N/F190N \\
\hline 1 & G359.797+0.037 & 266.247738 & -29.090525 & $8.715 \pm 0.023$ & $7.590 \pm 0.038$ & $7.030 \pm 0.013$ & $1088.79 \pm 17.10$ & $1.28 \pm 0.03$ \\
\hline 2 & G359.717-0.044 & 266.279460 & -29.200125 & $16.842 \pm 0.073$ & $13.526 \pm 0.019$ & $11.112 \pm 0.026$ & $12.58 \pm 0.20$ & $1.27 \pm 0.03$ \\
\hline 3 & G359.746-0.090 & 266.341231 & -29.199841 & $\cdots$ & $14.672 \pm 0.029$ & $12.703 \pm 0.028$ & $3.75 \pm 0.06$ & $1.88 \pm 0.05$ \\
\hline 4 & G359.691-0.072 & 266.290860 & -29.236897 & $15.555 \pm 0.030$ & $13.019 \pm 0.015$ & $11.140 \pm 0.018$ & $12.33 \pm 0.28$ & $1.48 \pm 0.05$ \\
\hline 5 & G359.907-0.001 & 266.350673 & -29.016080 & $\ldots$ & $13.454 \pm 0.033$ & $11.364 \pm 0.081$ & $103.74 \pm 1.63$ & $1.17 \pm 0.03$ \\
\hline 6 & G359.973-0.008 & 266.381294 & -28.954669 & $15.131 \pm 0.021$ & $12.677 \pm 0.012$ & $11.366 \pm 0.017$ & $15.28 \pm 0.24$ & $1.10 \pm 0.03$ \\
\hline 7 & G359.925-0.049 & 266.408263 & -29.026258 & $11.052 \pm 0.037$ & $9.578 \pm 0.044$ & $8.898 \pm 0.119$ & $185.76 \pm 2.92$ & $1.05 \pm 0.02$ \\
\hline 8 & G359.866-0.062 & 266.385493 & -29.082757 & $\cdots$ & $14.621 \pm 0.022$ & $12.016 \pm 0.014$ & $5.42 \pm 0.09$ & $1.80 \pm 0.04$ \\
\hline 9 & G0.070+0.025 & 266.422032 & -28.863311 & $14.799 \pm 0.016$ & $11.600 \pm 0.013$ & $9.858 \pm 0.011$ & $48.43 \pm 1.08$ & $1.19 \pm 0.04$ \\
\hline 10 & G0.058+0.014 & 266.426394 & -28.879828 & $14.704 \pm 0.016$ & $11.666 \pm 0.013$ & $10.110 \pm 0.028$ & $37.81 \pm 0.84$ & $1.33 \pm 0.04$ \\
\hline 11 & G0.114+0.021 & 266.452572 & -28.828510 & $\ldots$ & $13.612 \pm 0.025$ & $11.136 \pm 0.013$ & $12.57 \pm 0.20$ & $1.90 \pm 0.04$ \\
\hline 12 & G0.124+0.007 & 266.472510 & -28.827035 & $15.367 \pm 0.021$ & $12.535 \pm 0.017$ & $11.003 \pm 0.013$ & $19.20 \pm 0.30$ & $1.25 \pm 0.03$ \\
\hline 13 & G0.059-0.068 & 266.506989 & -28.920983 & $13.450 \pm 0.014$ & $10.687 \pm 0.011$ & $9.122 \pm 0.046$ & $109.86 \pm 1.73$ & $1.22 \pm 0.03$ \\
\hline 14 & G0.076-0.062 & 266.510914 & -28.903941 & $16.396 \pm 0.038$ & $13.478 \pm 0.017$ & $11.608 \pm 0.016$ & $8.67 \pm 0.16$ & $2.36 \pm 0.06$ \\
\hline 15 & G0.071-0.096 & 266.541810 & -28.925694 & $14.984 \pm 0.021$ & $12.273 \pm 0.017$ & $10.788 \pm 0.016$ & $22.50 \pm 0.35$ & $1.27 \pm 0.03$ \\
\hline 16 & G0.121-0.099 & 266.573243 & -28.884391 & $14.972 \pm 0.018$ & $12.053 \pm 0.014$ & $10.459 \pm 0.018$ & $29.98 \pm 0.47$ & $1.46 \pm 0.03$ \\
\hline 17 & G0.202-0.076 & 266.599325 & -28.803129 & $16.330 \pm 0.047$ & $13.265 \pm 0.011$ & $11.434 \pm 0.019$ & $11.68 \pm 0.18$ & $2.59 \pm 0.06$ \\
\hline 18 & G0.238-0.071 & 266.615120 & -28.770077 & $14.370 \pm 0.018$ & $11.291 \pm 0.012$ & $9.549 \pm 0.042$ & $69.06 \pm 1.09$ & $1.28 \pm 0.03$ \\
\hline 19 & G0.007-0.052 & 266.460700 & -28.957282 & $15.801 \pm 0.031$ & $12.933 \pm 0.031$ & $11.339 \pm 0.015$ & $13.05 \pm 0.21$ & $2.52 \pm 0.06$ \\
\hline$\ldots$ & G0.120-0.048 & 266.523436 & -28.858866 & $12.53 \pm 0.03$ & $9.24 \pm 0.02$ & $7.46 \pm 0.02$ & $918 \pm 14$ & $1.32 \pm 0.03$ \\
\hline
\end{tabular}

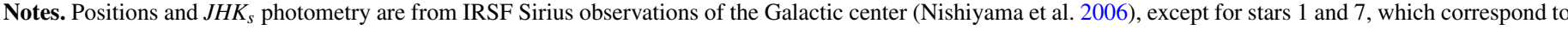

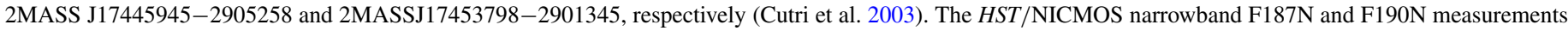
are from H. Dong et al. (2011, in preparation).

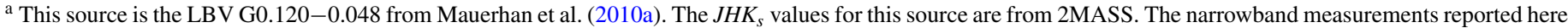

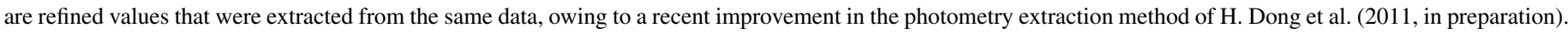

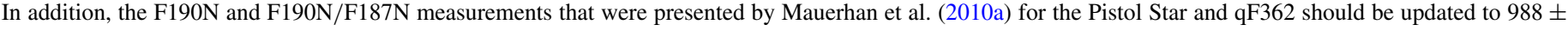
$18 \mathrm{mJy}$ and $1.21 \pm 0.03$ for the Pistol Star, and $740 \pm 13 \mathrm{mJy}$ and $1.02 \pm 0.03$ for $\mathrm{qF} 362$.

Narrowband photometry aimed at detecting emission-line excesses from hot supergiants can be very effective but requires a highly stable point-spread function (PSF) in order to accurately subtract the stellar continuum emission from the spectral line emission. This is difficult to achieve in seeing-limited conditions, especially when extreme stellar confusion is an issue, as it typically is when observing the GCR. Therefore, infrared narrowband surveys are best conducted from the stable environment of space, where low-background, diffraction-limited observations can be executed without the nuisance of PSF variability. Furthermore, space-based narrowband observations enable measurement of the Paschen- $\alpha(\mathrm{P} \alpha)$ transition $(n=4-3)$ of $\mathrm{HI}$, which is the strongest emission line of this species in the infrared. The $\lambda 1.87 \mu \mathrm{m}$ wavelength of this transition is also shared with several transitions of He I and He II, which is important in the interest of detecting hydrogen-poor WRs that exhibit strong helium emission.

In this work, we report discoveries of "isolated" emissionline stars in the GCR via $\mathrm{P} \alpha$ narrowband imaging from space and subsequent infrared spectroscopy from the ground. The new additions add to our recent identification of a new luminous blue variable in the GCR (Mauerhan et al. 2010a), which was the first reported discovery of the spectroscopic survey. The successful detection of these stars highlights the significant advantage that space-based narrowband searches using the $\mathrm{P} \alpha$ line have over ground-based surveys centered on other atomic transitions (e.g., Figer 1995; Homeier et al. 2003) which have identified only a fraction of the isolated emission-line stars. We present here the spectra of new identifications, their photometric properties, and discuss the implications that these objects have for massive star formation in the GCR.

\section{OBSERVATIONS}

\subsection{Selection of P $\alpha$ Emission-line Stars}

Our target sample was assembled following the completion of a $\mathrm{P} \alpha$ narrowband imaging survey of the GCR, performed with the Hubble Space Telescope and the Near-Infrared Camera and Multi-Object Spectrometer (NICMOS; Wang et al. 2010). The survey, requiring 144 orbits of observations, covered the central $(l, b) \approx 39 \times 15 \operatorname{arcmin}^{2}$ around Sgr A*, which corresponds to a total projected physical area of $\approx 90 \times 35 \mathrm{pc}^{2}$, assuming a distance of $8 \mathrm{kpc}$ to Sgr A* (Reid 1993 and references therein). The NIC3 camera was utilized and narrowband images were obtained through the F187N (line; $\lambda=1.874 \mu \mathrm{m}$; FWHM $=$ $0.019 \mu \mathrm{m}$ ) and F190N (continuum; $\lambda=1.901 \mu \mathrm{m} ; \mathrm{FWHM}=$ $0.017 \mu \mathrm{m}$ ) filters. The survey resulted in the detection of $\approx 150$ point-like sources of $\mathrm{P} \alpha$ line excess (i.e., sources with F187N-F190N values $\gtrsim 5 \sigma$ above the mean of all point sources in the survey area; H. Dong et al. 2011, in preparation). Approximately half of the sources are located outside of the three known stellar clusters, and nearly half of those were unidentified prior to this work.

We have undertaken a near-infrared spectroscopic campaign to characterize the unidentified sources of $\mathrm{P} \alpha$ line excess. Focusing on the sources having the strongest line excess and the brightest near-infrared counterparts ( $K_{s} \lesssim 12.7 \mathrm{mag}$ ), we have spectroscopically identified 19 new, hot, massive stars in the GCR. So far, our spectroscopic search has had a $100 \%$ success rate for detecting emission-line stars. Table 1 lists basic data for these sources, including their positions, corresponding $J H K_{S}$ photometry, and narrowband F187N and F190N measurements. The sources are named based on their Galactic coordinates, 
but will hereafter be referred to by their numerical entry in Table 1 . The positions and $J H K_{s}$ photometry were taken from the catalog of the Sirius survey of the GCR (see Nishiyama et al. 2006 and references therein), which generated a catalog of point sources having a $10 \sigma$ limiting $K_{s}$ magnitude of 15.6. The F187N and F190N measurements are from H. Dong et al. (2011, in preparation). We note that the method of extraction for the narrowband photometry has been refined since our report on the LBV G0.120-0.048 (Mauerhan et al. 2010a), where we presented our narrowband measurements for this source and the known LBVs qF362 (aka FMM 362; Geballe et al. 2000) and the Pistol Star (Figer et al. 1998); so, we take this opportunity to include the refined narrowband measurements for all three of these LBVs in Table 1.

\subsection{Spectroscopic Observations}

Spectra of four stars were obtained using the SpeX mediumresolution spectrograph on the $3 \mathrm{~m}$ Infrared Telescope Facility (IRTF) telescope (Rayner et al. 2003), located on the summit of Mauna Kea in Hawaii. SpeX was used in short crossdispersed mode (SXD), utilizing a slit width of 0'.5, providing a spectral resolution of $R=\lambda / \delta \lambda \approx 1200$. Flat-field images and a wavelength calibration source were provided by obtaining spectra of continuum and argon lamp sources. The data were reduced and extracted using the IDL-based software package Spextool, specially designed for the reduction of data obtained with SpeX on the IRTF (Cushing et al. 2004).

The $3 \mathrm{~m}$ United Kingdom Infrared Telescope (UKIRT) on Mauna Kea was used to obtain spectra of five stars. The UKIRT 1-5 $\mu \mathrm{m}$ Imager Spectrometer (UIST; Ramsay Howatt et al. 2004) was used in service mode as part of the UKIRT Service Programme. The short- $K$ grism and 4 pixel slit $\left(00^{\prime} 48\right)$ were used, providing a spectral resolution of $R \approx 2133$ and a wavelength range of $\lambda 2.01-2.26 \mu \mathrm{m}$. Flat fields and a wavelength calibration source were provided by obtaining spectra of continuum and argon lamps. The data images were reduced using the Starlink ORACDR pipeline. The spectra were extracted using the IRAF routine APALL.

Spectra of four stars were obtained using the $4.1 \mathrm{~m}$ AngloAustralian Telescope (AAT) on Siding Spring Mountain (Mount Woorat) in New South Wales, Australia. The IRIS2 instrument (Tinney et al. 2004) provided a spectral resolution of $R \approx$ 2400 in the $K$ band, using the $1^{\prime \prime}$ slit. Flat-field images and a wavelength calibration source were provided by obtaining spectra of continuum and neon lamp sources. The data images were reduced and spectra extracted with the same programs used for the UKIRT data.

Spectra of six stars were obtained at the Southern Observatory for Astrophysical Research (SOAR), located on Cerro Pachon in Chile. The Ohio State Infrared Imager Spectrometer (OSIRIS; Depoy et al. 1993) was used in cross-dispersed mode for five of these stars. This mode provides a spectral resolution of $R \approx 1200$. Wavelength calibration was achieved using the background $\mathrm{OH}$ emission lines from the sky. For one star, OSIRIS was used in the higher resolution long-slit mode to obtain a $K$-band spectrum with a resolution $R \approx 3000$. For this observation, wavelength calibration was performed using spectra of the internal neon and argon lamps. All of these data were reduced and extracted using the standard IRAF routines and APALL.

For all observations the spectra were acquired in an "ABBA" nodding sequence in order to subtract the sky background, and to suppress the contribution of bad pixels. Spectra of A0V standard stars were obtained at air masses similar to those of the
Table 2

Spectroscopic Observations

\begin{tabular}{cccc}
\hline \hline Star & Observation Date (UT) & Telescope/Instrument & $\lambda / \delta \lambda$ \\
\hline 1 & 2009 Aug 4 07:31 & IRTF/SpeX & 2000 \\
2 & 2009 Jun 13 07:20 & SOAR/OSIRIS & 1200 \\
3 & 2009 Aug 4 08:06 & IRTF/SpeX & 1200 \\
4 & 2009 Jun 13 05:29 & SOAR/OSIRIS & 1200 \\
5 & 2007 Aug 1 08:30 & UKIRT/UIST & 2133 \\
6 & 2010 May 24 06:52 & SOAR/OSIRIS & 1200 \\
7 & 2010 May 27 10:23 & SOAR/OSIRIS & 1200 \\
8 & 2009 Aug 4 07:42 & IRTF/SpeX & 1200 \\
9 & 2007 Aug 1 09:05 & UKIRT/UIST & 2133 \\
10 & 2007 Aug 1 06:36 & UKIRT/UIST & 2133 \\
11 & 2008 Jul 31 09:10 & 2133 \\
12 & 2007 Aug 1 05:57 & UKIRT/UIST & 2133 \\
13 & 2008 Jun 17 09:19 & SOAR/OSIRIS & 3000 \\
14 & 2008 May 16 14:00 & AAT/IRIS2 & 2400 \\
15 & 2009 Aug 4 08:40 & IRTF/SpeX & 1200 \\
16 & 2008 May 14 19:37 & AAT/IRIS2 & 2400 \\
17 & 2008 May 16 15:45 & AAT/IRIS2 & 2400 \\
18 & 2008 Jun 13 01:10 & SOAR/OSIRIS & 1200 \\
19 & 2009 May 16 13:41 & AAT/IRIS2 & 2400 \\
\hline
\end{tabular}

science targets in order to derive a telluric absorption spectrum. The telluric corrections were applied using the IDL package xtellcor (Vacca et al. 2003), which removes model Hi absorption lines from the A0V standard star before application to the science data. Table 2 summarizes the facilities used for the observations of each star.

\section{SPECTROSCOPIC CLASSIFICATION}

In the following sections, the spectroscopic criteria we use to classify these stars were adopted from previous spectroscopic studies of massive stars in the near-infrared, conducted by Morris et al. (1996), Figer et al. (1997), and Martins et al. (2008). The central wavelengths of all spectral lines referred to in the text are adopted from these references.

\subsection{Early O Supergiants and Weak-lined WNh Stars}

The $K$-band spectra of stars 6 and 7 are presented in Figure 1 . The spectra are dominated by a complex of blended emission lines near $\lambda 2.112-2.115 \mu \mathrm{m}$, which includes contributions from He I, N III, C III, and O III. Blueward of this line complex are emission lines of $\mathrm{C}_{\mathrm{IV}}$ at $\lambda 2.069$ and $\lambda 2.078 \mu \mathrm{m}$. Br $\gamma$ emission is marginally detected in the spectrum of star 6 , although it may be a background nebular feature. The spectra of both stars exhibit He II absorption at $\lambda 2.189 \mu \mathrm{m}$, although it is very near the noise level. These features are consistent with the $K$-band spectra of early $\mathrm{O}$ supergiants, specifically in the range of O4-6I (e.g., see Martins et al. 2008).

The spectra of stars $16,15,12,9$, and 10 are presented in Figure 2. The spectra of these stars are dominated by emission lines of $\mathrm{Br} \gamma$ and the $\lambda 2.112-2.115 \mu \mathrm{m}$ complex of He I, N III, C III, and O III. Stars 16, 15, and 12 exhibit the $\lambda 2.112-2.115 \mu \mathrm{m}$ complex in fairly strong emission, while 9 and 10, instead, exhibit a He I absorption line accompanied by a relatively weak emission component. Weak C IV emission is also apparent in the spectra of stars 16,15 , and 12 . The $\mathrm{Br} \gamma$ lines of all of these stars exhibit an asymmetry on their blue side owing to a contribution from $\mathrm{He}_{\mathrm{I}}$ at $\lambda 2.1647 \mu \mathrm{m}$. An absorption line of $\mathrm{He}_{\mathrm{I}}$ at $\lambda 2.058 \mu \mathrm{m}$ is present for four of the stars, while this feature appears in emission for two of them. Weak N III emission appears at $\lambda 2.247 \mu \mathrm{m}$ in the spectrum of star 16 . Three 


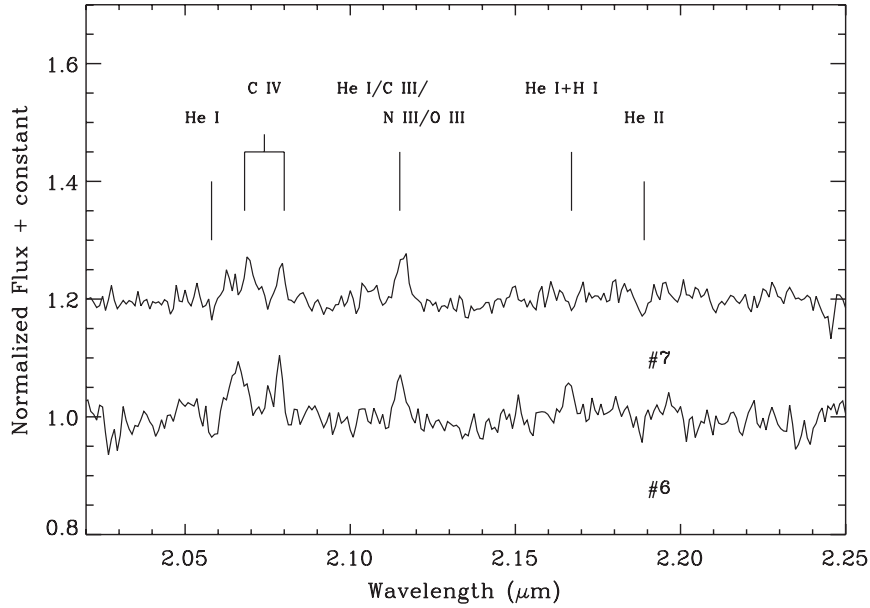

Figure 1. $K$-band spectra of confirmed O4-6I stars G359.973-0.008 (star 6) and G359.925-0.049 (star 7), which are counterparts to Chandra X-ray sources CXOGC J174531.4-285716 and CXOGC J174537.9-290134, respectively.

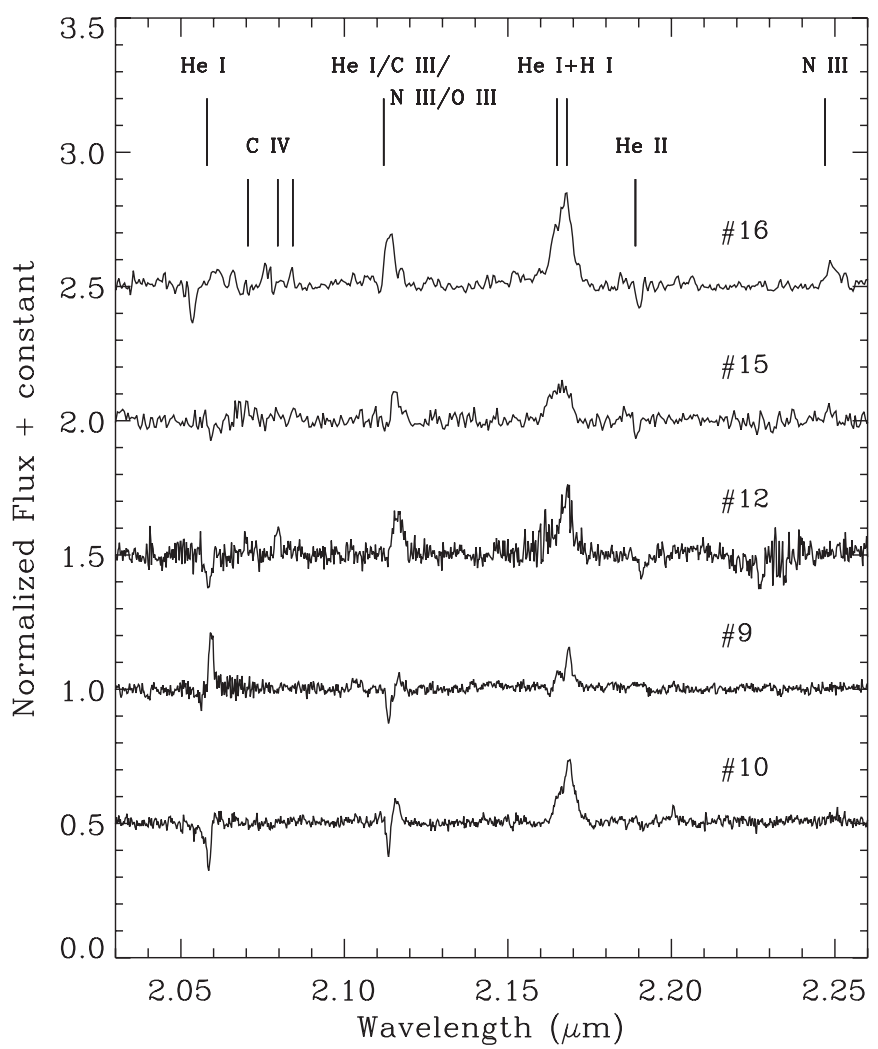

Figure 2. $K$-band spectra of confirmed $\mathrm{WNh}$ and $\mathrm{O} 4-6 \mathrm{If}^{+}$stars.

of the stars also exhibit weak He II absorption at $\lambda 2.189 \mu \mathrm{m}$, while this transition is not detected in the spectra stars 9 and 10. These features are characteristics of $\mathrm{OIf}^{+6}$ supergiants and late-type WN stars (WNL), which are commonly referred to as hydrogen-rich WRs (WNh stars). Distinguishing between these two types can be very difficult indeed. According to Martins et al. (2008), OIf $^{+}$stars exhibit $\mathrm{Br} \gamma$ emission that has approximately equivalent strength to the $\lambda 2.112-2.115 \mu \mathrm{m}$ complex, while WN8-9h stars exhibit a relatively dominant $\mathrm{Br} \gamma$ emission line. Based on the dominance of $\mathrm{Br} \gamma$ in the spectra of all stars in Figure 2, we may be inclined to classify them all as

6 Historically, the "+" in the $\mathrm{OIf}^{+}$designation is given to $\mathrm{O}$ stars exhibiting S IV emission ( $\lambda \lambda 4089$ and 4116$)$, as well as $\mathrm{N}$ III and He II $\lambda 4686$ emission in their optical spectra (Walborn 1971).

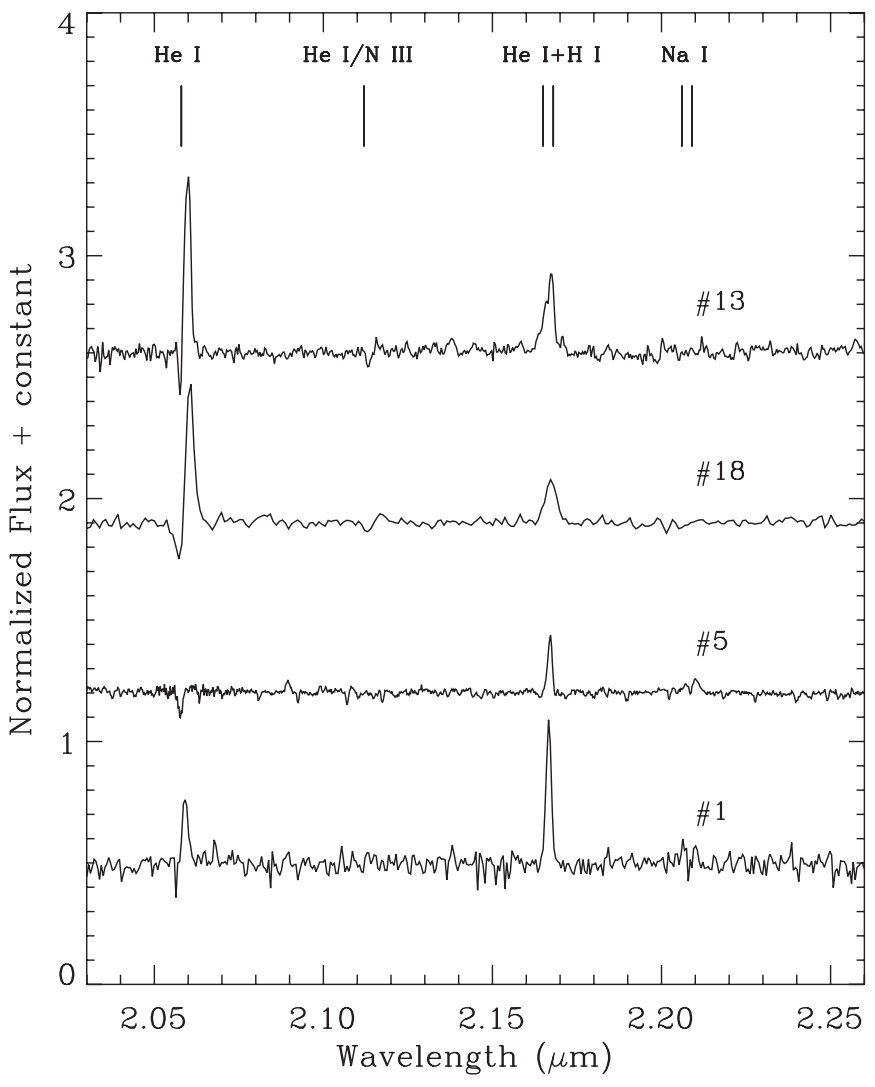

Figure 3. $K$-band spectra of $\mathrm{P}$ Cygni-type $\mathrm{O}$ supergiants and B supergiants.

WN8-9h stars. However, the weakness of the $\lambda 2.112-2.115 \mu \mathrm{m}$ complex in the spectra of stars 9 and 10 is more typical of the O4-6If ${ }^{+}$stars in Martins et al. (2008) than the WN8-9h stars, which always have a strong $\lambda 2.112-2.115 \mu \mathrm{m}$ emission complex. Furthermore, WN8-9h always exhibit He II emission or absorption at $\lambda 2.189 \mu \mathrm{m}$, but this transition is not detected in the spectra of stars 9 and 10 . Thus, we classify stars 16,15 , and 12 as WN8-9h stars, and assign the O4-6If ${ }^{+}$designation to stars 9 and 10.

\subsection{Other OB Supergiants}

The spectra of stars 13, 18, 5, and 1 are presented in Figure 3. All of these stars exhibit prominent $\mathrm{Br} \gamma$ emission. Stars 13 and 18 also exhibit strong He I emission at $\lambda 2.058 \mu \mathrm{m}$ with a P Cygni profile, and weak He I absorption at $\lambda 2.112 \mu \mathrm{m}$ that may also include a weak $\mathrm{N}$ III emission component on the red side of the He I absorption line. The $\mathrm{Br} \gamma$ line of star 13 exhibits an asymmetry on its blue side owing to a contribution from He I. Star 1 exhibits He I at $\lambda 2.058 \mu \mathrm{m}$ in emission, although it is relatively weak. Star 5 exhibits the He I $\lambda 2.058 \mu \mathrm{m}$ feature as well, but it is in weak absorption. The spectra of stars 5 and 1 exhibit weak emission from the Na I doublet near $\lambda 2.209 \mu \mathrm{m}$, and in the case of star 5, weak Fe II emission near $\lambda 2.09 \mu \mathrm{m}$. The presence of these low-ionization emission features indicates that stars 1 and 5 are probably of the cooler B spectral class, with subtypes in the range of B0-B2 (e.g., see Hanson et al. 1996). Their classification as supergiants is justified in Section 4.2. By comparison, stars 13 and 18 must be hotter, as indicated by the presence of He I absorption, while the strong P Cygni emission lines of $\mathrm{He}$ I at $\lambda 2.058 \mu \mathrm{m}$ indicate relatively strong winds. Based on these features, we conclude that stars 13 and 18 should be classified as $\mathrm{P}$ Cygni-type $\mathrm{O}$ supergiants. 


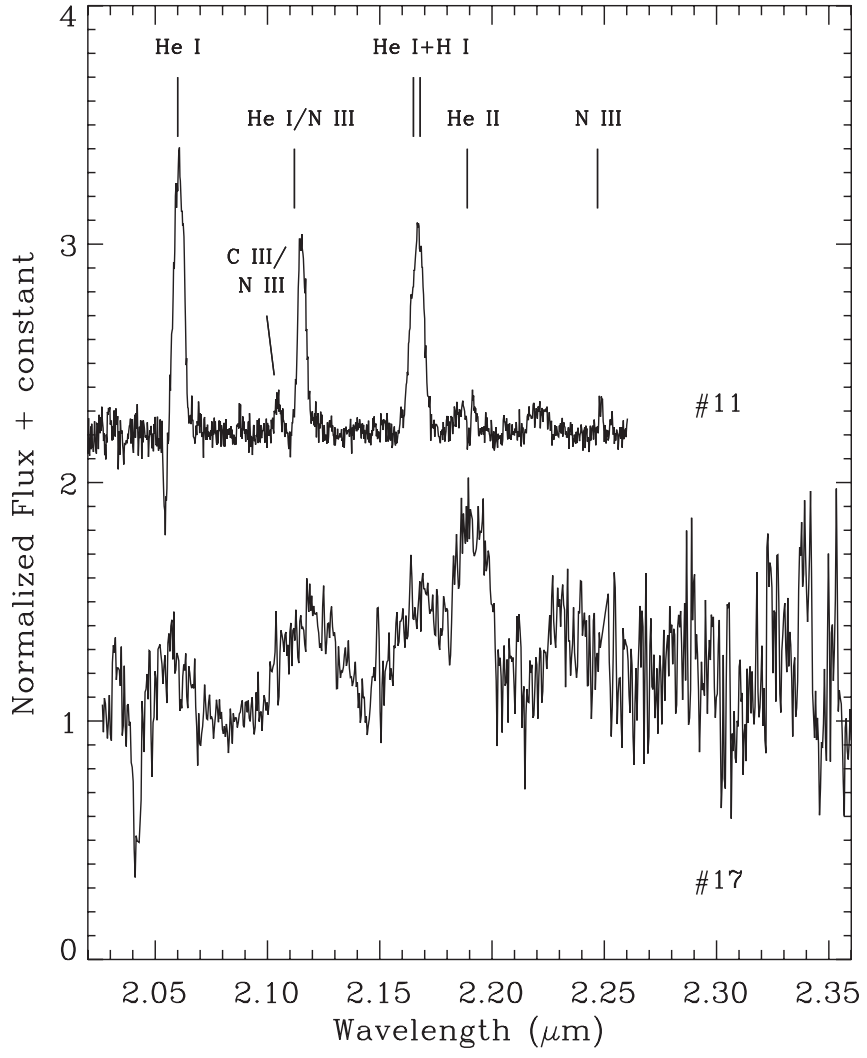

Figure 4. $K$-band spectra of confirmed strong-lined $\mathrm{WN}$ stars.

\subsection{Strong-lined WN Stars}

Figure 4 presents spectra of stars 11 and 17. These stars exhibit broad emission lines, indicative of fast, extended stellar winds. For star 11 spectral emission features include $\mathrm{Br} \gamma$, $\mathrm{He}$ I at $\lambda 2.058 \mu \mathrm{m}$ and $\lambda 2.112 \mu \mathrm{m}$, and $\mathrm{He}$ II at $\lambda 2.189 \mu \mathrm{m}$. The latter of these features is probably the superposition of an He II $\lambda 2.189 \mu \mathrm{m}$ P Cygni profile and a blend of He I emission at $\lambda 2.185 \mu \mathrm{m}$. Weak emission from $\mathrm{N}_{\text {III }}$ is also present at $\lambda 2.247 \mu \mathrm{m}$, as well as a weak C III/N III blend at $\lambda 2.104 \mu \mathrm{m}$. The weakness of $\mathrm{He}$ II relative to $\mathrm{Br} \gamma$ and $\mathrm{He}$ I at $\lambda 2.112 \mu \mathrm{m}$, and the strong P Cygni emission component of $\mathrm{He}$ I at $\lambda 2.058 \mu \mathrm{m}$, are characteristics of late-type, hydrogen-rich, nitrogen-type stars, specifically WN8-9h stars (Figer et al. 1997; Martins et al. 2008).

The dominant emission feature in the spectrum of star 17 is He II at $\lambda 2.189 \mu \mathrm{m}$, which indicates that it is of an early WN (WNE) subtype. Weak but broad He I emission at $\lambda 2.058 \mu \mathrm{m}$ is present with a deep and highly blueshifted P Cygni absorption component. The large blueshift of this absorption component relative to the peak of the emission component highlights the high wind velocity of star $17(\delta \lambda=0.015 \mu \mathrm{m} \Rightarrow$ $\left.v_{\text {wind }} \approx 2200 \mathrm{~km} \mathrm{~s}^{-1}\right)$ relative to star $11(\delta \lambda=0.006 \mu \mathrm{m} \Rightarrow$ $v_{\text {wind }} \approx 900 \mathrm{~km} \mathrm{~s}^{-1}$ ). Furthermore, the emission lines of star 17 are exceptionally broad, having FWHM $=155 \AA$. According to Crowther et al. (2006), WN stars with FWHM(He II $\lambda 2.189 \mu \mathrm{m}) \geqslant 130 \AA$ are classified as broad-lined WN stars (WNb). Star 17 satisfies the broad criterion, while the relative strength of $\mathrm{He}$ II relative to $\mathrm{Br} \gamma$ and $\mathrm{He} \mathrm{I}$ is consistent with a WN5 spectral type. Thus, we classify star 17 as a WN5b star.

\subsection{WC Stars}

Figure 5 presents the $K$-band spectra of star $8,19,14,3$, 2 , and 4 . Each of these stars exhibit emission from $\mathrm{C}_{\text {III }}$ at

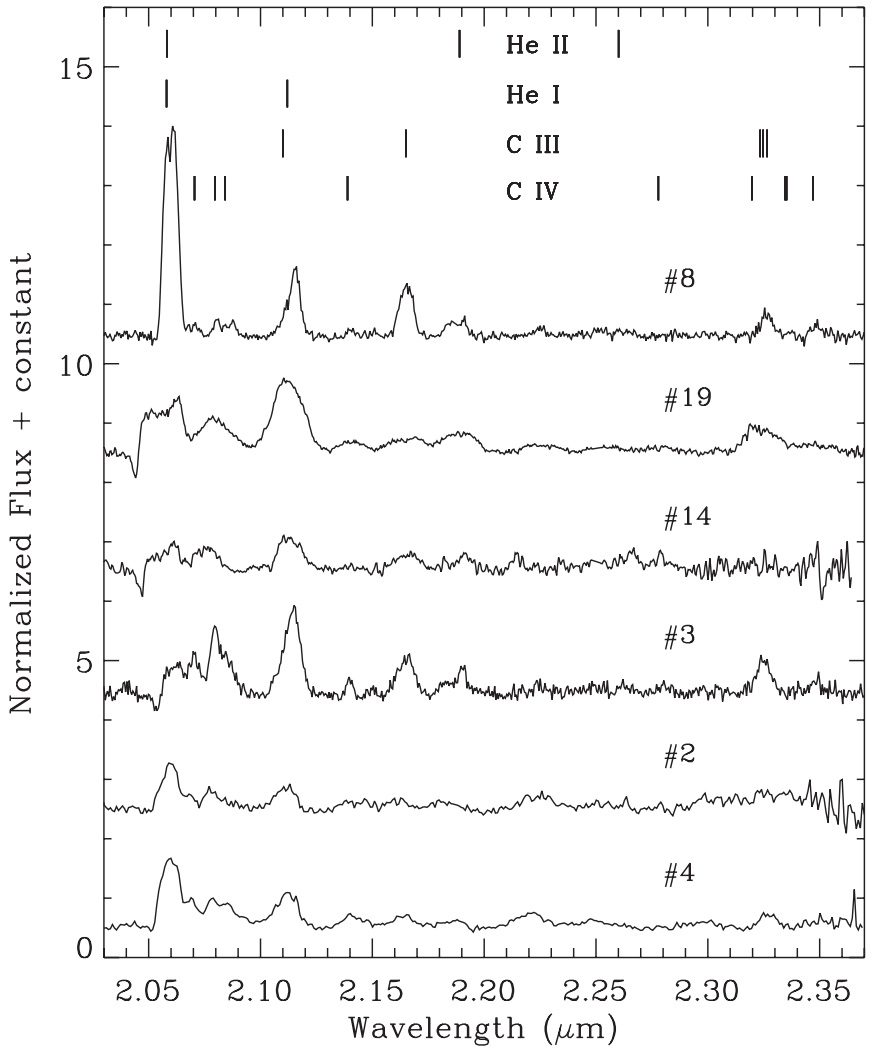

Figure 5. $K$-band spectra of confirmed WC9 stars

$\lambda 2.115 \mu \mathrm{m}, \mathrm{He}$ I, and He II near $\lambda 2.058 \mu \mathrm{m}, \mathrm{C}$ III, and C IV near $\lambda 2.07-2.08 \mu \mathrm{m}$, and weak He II emission near $\lambda 2.189 \mu \mathrm{m}$. These spectral characteristics are consistent with those of late, carbon-type (WC) WRs. A subtype diagnostic is provided by the strength ratio of $\mathrm{C}_{\text {IV }}$ at $\lambda 2.08 \mu \mathrm{m}$ relative to $\mathrm{C}_{\text {III }}$ at $\lambda 2.115 \mu \mathrm{m}$ (Figer et al. 1997), which is less than unity for all stars, consistent with WC9 subtype.

\section{PHOTOMETRIC PROPERTIES}

\subsection{Infrared Excess}

Luminous WR and $\mathrm{O}$ stars generally exhibit infrared excess from the free-free emission generated in their ionized winds, and also from strong line emission (Hadfield et al. 2007; Mauerhan et al. 2009b). Thermal continuum excess from hot dust emission is also not uncommon to late-type WC (WCL) stars. Color-color diagrams are useful in assessing the infrared excess of WRs and the likely causes for it. Figure 6 shows a color-color diagram of $H-K_{s}$ versus $J-H$ for the stars in our sample that were detected in all three near-infrared bands. The interstellar reddening vector is illustrated by the linear trend traced by random field stars that lie toward the GCR, most of which are presumably late-type giants. Stars 1 and 7 lie near the lower left of Figure 6, well separated from most of the other massive stars. The fact that they suffer relatively low extinction implies that they are in the foreground toward the GCR. They also do not appear to exhibit evidence for significant, intrinsic excess.

While the OB and WN stars in the GCR have color excesses typical for their class, several WC stars in Figure 6 appear to have color excesses that are significantly higher than those of the $\mathrm{O}$ and WN stars and $1 \mathrm{WC}$ star (note that only four of the WC stars from our sample are included in Figure 6, since two others 
Table 3

Extinction and Absolute Photometry for Selected Stars

\begin{tabular}{|c|c|c|c|c|c|c|c|c|c|c|}
\hline Star & $\begin{array}{l}\text { Spectral } \\
\text { Type }\end{array}$ & $\begin{array}{c}K_{S} \\
(\mathrm{mag})\end{array}$ & $\begin{array}{c}\left(J-K_{s}\right)_{0} \\
(\mathrm{mag})\end{array}$ & $\begin{array}{c}\left(H-K_{s}\right)_{0} \\
(\mathrm{mag})\end{array}$ & $\begin{array}{c}A_{K_{S}}^{J-K_{S}} \\
(\mathrm{mag})\end{array}$ & $\begin{array}{c}A_{K_{s}}^{H-K_{s}} \\
(\mathrm{mag})\end{array}$ & $\begin{array}{c}\overline{A_{K_{S}}} \\
(\mathrm{mag})\end{array}$ & $\begin{array}{c}M_{K_{s}} \\
\text { (mag) }\end{array}$ & $\begin{array}{l}\mathrm{BC}_{K}^{\mathrm{a}} \\
(\mathrm{mag})\end{array}$ & $\begin{array}{c}L_{\text {bol }} \\
\left(\log L_{\odot}\right)\end{array}$ \\
\hline 1 & B0I-B2I & 7.03 & -0.21 & -0.10 & 0.94 & 0.96 & $0.95^{\mathrm{b}}$ & $-6.71^{b}$ & -3.7 & $6.06^{\mathrm{b}}$ \\
\hline 2 & WC9?d & 11.11 & 0.23 & 0.26 & 2.91 & 2.86 & 2.89 & -6.28 & -3.6 & $5.85^{\mathrm{b}}$ \\
\hline 3 & WC9 & 12.70 & 0.23 & 0.26 & $\ldots$ & 4.06 & 4.06 & -5.86 & -3.6 & 5.68 \\
\hline 4 & WC9?d & 11.14 & 0.23 & 0.26 & 2.21 & 2.15 & 2.18 & -5.54 & -3.6 & $5.56^{\mathrm{c}}$ \\
\hline 5 & B0I-B2I & 11.36 & -0.21 & -0.10 & $\cdots$ & 3.16 & 3.16 & -6.30 & -3.7 & 5.90 \\
\hline 6 & O4-6I & 11.37 & -0.21 & -0.10 & 1.96 & 2.05 & 2.01 & -5.14 & -4.3 & 5.68 \\
\hline 7 & O4-6I & 8.90 & -0.21 & -0.10 & 1.17 & 1.14 & $1.16^{\mathrm{b}}$ & $-5.14^{\mathrm{b}}$ & -4.3 & $5.68^{b}$ \\
\hline 8 & WC9 & 12.02 & 0.23 & 0.26 & $\ldots$ & 3.12 & 3.12 & -5.60 & -3.6 & 5.58 \\
\hline 9 & O4-6 If $^{+}$ & 9.86 & -0.21 & -0.10 & 2.54 & 2.67 & 2.61 & -7.25 & -4.3 & 6.52 \\
\hline 10 & O4-6 $\mathrm{If}^{+}$ & 10.11 & -0.21 & -0.10 & 2.37 & 2.40 & 2.39 & -6.78 & -4.3 & 6.33 \\
\hline 11 & WN8-9h & 11.14 & 0.13 & 0.11 & $\ldots$ & 3.41 & 3.41 & -6.77 & -4.2 & 6.29 \\
\hline 12 & WN8-9h & 11.00 & 0.13 & 0.11 & 2.09 & 2.05 & 2.07 & -5.57 & -4.2 & 5.81 \\
\hline 13 & P Cyg-type OI & 9.12 & -0.21 & -0.10 & 2.24 & 2.41 & 2.33 & -7.71 & -3.7 & 6.46 \\
\hline 14 & WC9 & 11.61 & 0.23 & 0.26 & 2.41 & 2.14 & 2.28 & -5.17 & -3.6 & 5.41 \\
\hline 15 & WN8-9h & 10.79 & 0.13 & 0.11 & 2.01 & 1.98 & 2.00 & -5.71 & -4.2 & 5.86 \\
\hline 16 & WN8-9h & 10.46 & 0.13 & 0.11 & 2.17 & 2.24 & 2.15 & -6.19 & -4.2 & 6.06 \\
\hline 17 & WN5b & 11.43 & 0.36 & 0.26 & 2.40 & 2.09 & 2.24 & -5.31 & -4.4 & 5.54 \\
\hline 18 & P Cyg-type OI & 9.55 & -0.21 & -0.10 & 3.37 & 3.35 & 3.36 & -8.31 & -3.7 & 6.70 \\
\hline 19 & WC9 & 11.34 & 0.23 & 0.26 & 2.10 & 1.92 & 2.01 & -5.17 & -3.6 & 5.41 \\
\hline
\end{tabular}

Notes.

a Bolometric corrections were derived from Martins et al. (2008) for WN8-9h stars; from Crowther et al. (2006) for other WRs; and from Martins \& Plez (2006) for the OB stars.

b The relatively low value of extinction for the O4-6I star 7 and B0I-B2I star 1, compared to the other stars in this table, implies that they are located in the foreground. We estimated the distance to 7 (O4-6I) by adopting $M_{K_{s}}$ and $L_{\text {bol }}$ from the similar O4-6I star 6. The resulting distance modulus is $12.79 \mathrm{mag}(3.6 \mathrm{kpc})$, which implies that 7 lies in the Norma arm of the Galaxy (Churchwell et al. 2009). Since star 1 has an extinction that is comparable to 7 , one might also assume that these stars have comparable distances. If so, this implies 1 has $M_{K_{s}}=-6.71 \mathrm{mag}$, which means it is a supergiant.

${ }^{c}$ These values may be erroneously high. The color-color diagram in Figure 6 implies that these stars may emit thermal excess from hot dust, in which case the extinction cannot be reliably derived using the near-infrared colors.

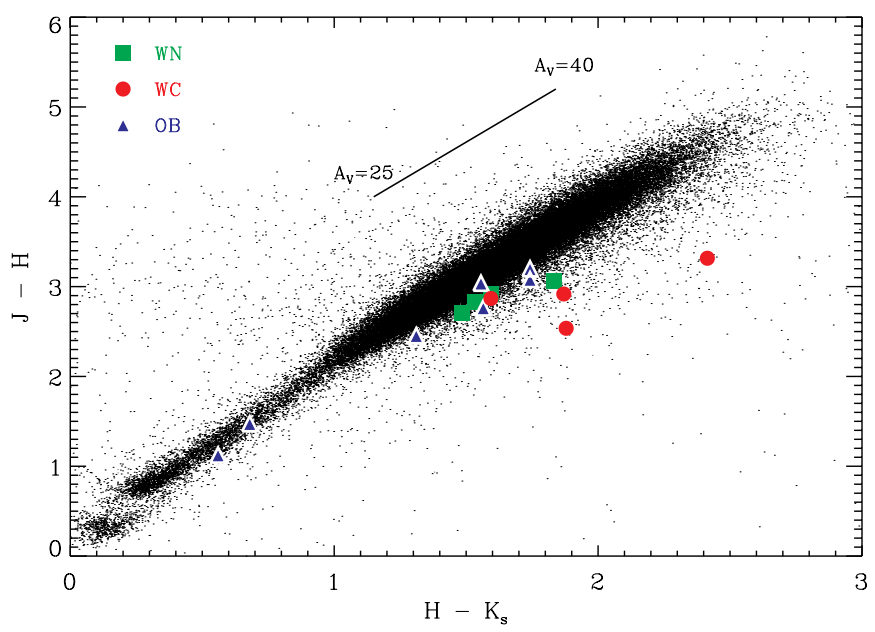

Figure 6. Near-infrared color-color diagram of new WN, WC, and OB stars (green squares, red circles, and blue triangles, respectively). The small dots are random field stars. All of the massive stars exhibit infrared excess, mainly attributable to free-free and line emission from their dense, ionized winds. The two WC9 stars farthest from the locus of field stars, 2 and 4, may be sources of an additional excess component of thermal emission from hot dust.

(A color version of this figure is available in the online journal.)

have not been detected in the $J$ band). The colors of star 19 are consistent with the excess being from free-free emission alone, and this might also be true for star 14 . However, the relatively strong excess emission from stars 2 and 4 (the two WC stars that are furthest from the reddening trend in Figure 6) indicates that there is an additional excess component, which is likely the result of thermal emission from hot dust. Late-type WC stars (WC8-9) commonly exhibit thermal emission from hot dust, and it is generally accepted that the production of dust from these stars is a consequence of binary evolution, whereby the dust is created via the collision of hydrogen-rich and carbonrich winds from the respective OB and WC stars (Williams et al. 2005). Several likely examples of this phenomenon are already known in the GCR, and they are typically detectable as hard $\mathrm{X}$-ray sources (e.g., see Mauerhan et al. 2010b and references therein). Since stars 2 and 4 were not detected in X-ray surveys of their respective fields (Muno et al. 2009), there is currently no evidence that they are members of colliding-wind binary systems. Nonetheless, the relatively strong excess emission from stars 2 and 4 is consistent with thermal dust emission. Thus, we tentatively classify stars 2 and 4 as WC9?d stars.

\subsection{Extinction and Luminosity}

We derived the extinction and absolute photometry for each source by comparing the observed $J-K_{s}$ and $H-K_{s}$ colors with the intrinsic colors of stars of the same spectral types, which were adopted from the literature (Martins \& Plez 2006; Crowther et al. 2006). We used the extinction relation of Nishiyama et al. (2006) for stars near the Galactic center, which is given by

$$
A_{K_{s}}=1.44 \pm 0.01 E_{H-K_{s}}
$$


and

$$
A_{K_{s}}=0.494 \pm 0.006 E_{J-K_{s}},
$$

where $E\left(H-K_{s}\right)=\left(H-K_{s}\right)_{\text {obs }}-\left(H-K_{s}\right)_{0}$, and similarly for the $J-K_{s}$ colors. The two values were then averaged to obtain a final extinction estimate, $\overline{A_{K_{s}}}$. The results are listed in Table 3. The values are similar to other known massive stars in the GCR (e.g., see Mauerhan et al. 2010b). As expected from their positions in Figure 6, stars 7 (O4-6I) and 1 (B0I-B2I) have relatively low $A_{K_{s}}$ values of 1.16 and $0.95 \mathrm{mag}$, respectively, which indicates that they lie in the foreground.

Assuming a distance of $8 \mathrm{kpc}$ for the Galactic center (Reid 1993), we derived $M_{K_{s}}$ from the extinction-corrected values. To calculate the bolometric luminosities of the stars, bolometric corrections for the appropriate spectral types were adopted from the same literature sources used to obtain the intrinsic colors, except for the WN8-9h stars, whose bolometric corrections were derived from the $M_{K}$ and luminosity values of the WN8-9h stars in Martins et al. (2008). These results are also listed in Table 3, and they are more or less consistent with luminosities that are typical for these spectral types. The luminosity values derived for the other $\mathrm{O}$ and $\mathrm{B}$ stars are also consistent with supergiant luminosity class. We note that the luminosity values obtained for WC9?d stars should be met with caution, since thermal dust emission can have a significant effect on the observed infrared colors, leading to an overestimate of the extinction toward the source. Nonetheless, the luminosity values that we obtain are consistent with those of other WC9 stars. For the foreground O-4I star 7, we assumed that it shares the same value of $M_{K}$ as star 6 , since they appear to be identical in spectral type. Thus, we derive a distance modulus of $12.79 \mathrm{mag}$ $(3.6 \mathrm{kpc})$ to star 7, which places it in the Norma arm of the Galaxy (Churchwell et al. 2009). For the B0I-B2I star 1 we have no information on its luminosity class, so we are unable to derive its foreground distance. However, its extinction value is very comparable to star 7; so, we could infer that it also lies at roughly the same distance, also in the Norma arm. If so, then the associated distance modulus of 12.79 mag indicates that star 1 has $M_{K_{s}}=-6.71$, which implies supergiant luminosity class.

\section{X-RAY DETECTIONS}

The O4-6I stars 6 and 7 have X-ray counterparts in the master catalog of Muno et al. (2009), which contains 9017 X-ray point sources detected in all combined observations of the GCR with the Chandra X-ray Observatory. The $\mathrm{X}$-ray photometric data for these two sources is presented in Table 4. The details of the extraction process are described in Muno et al. (2009). Candidate near-infrared counterparts to the population of GCR X-ray sources were identified in Mauerhan et al. (2009a); the O4-6I star 6 is source 432 from Table 3 of those authors, and is associated with the Chandra source CXOGC J174531.4-285716. On the other hand, the foreground O4-6I star 7 that is associated with Chandra source CXOGC J174537.9-290134 is not present in Mauerhan et al. (2009a), since it was flagged as having erroneous photometry in the Sirius near-infrared catalog that was used for that work; so, for this star we relied on Two Micron All Sky Survey (2MASS) photometry. Table 4 includes X-ray source astrometry, total on-source integration time, photon number counts in the hard $(2.0-8.0 \mathrm{keV})$ and soft bands (0.5-2.0 keV), broadband photon flux, hardness ratios for soft and hard energy bands, and the average energy of all detected photons.
Table 4 Chandra X-ray Data for G359.973-0.008 and G359.925-0.049

\begin{tabular}{lcc}
\hline \multicolumn{1}{c}{ Value } & G359.973-0.008 (6) & G359.925-0.049 (7) \\
\hline Spectral type & O4-6I & O4-6I \\
CXOGC J & $174531.4-285716$ & $174537.9-290134$ \\
R.A. (J2000) & 266.38108 & 266.40829 \\
Decl. $(\mathrm{J} 2000)$ & -28.95466 & -29.02625 \\
$\sigma_{X}(\operatorname{arcsec})$ & 0.5 & 0.3 \\
$C_{\text {net }}^{\text {soft }}(0.5-2.0 \mathrm{keV})$ & $10.8_{-7.3}^{+9.5}$ & $140.6_{19.2}^{19.9}$ \\
$C_{\text {net }}^{\text {hard }}(2.0-8.0 \mathrm{keV})$ & $58.8_{-23.0}^{+17.3}$ & $150.8_{-21.2}^{+21.4}$ \\
$F_{\text {tot }}\left(\mathrm{s}^{-1} \mathrm{~cm}^{-2}\right)$ & $2.44 \times 10^{-7}$ & $1.37 \times 10^{-6}$ \\
$\mathrm{HR} 0$ & $0.57_{-0.31}^{+0.28}$ & $-0.17_{-0.10}^{+0.11}$ \\
$\mathrm{HR} 2$ & -1.00 & $-0.07_{-0.26}^{+0.26}$ \\
$\langle E\rangle /$ photon $(\mathrm{keV})$ & 3.0 & 2.5 \\
$k T(\mathrm{keV})$ & $0.6-1.0$ & $0.9 \pm 0.2$ \\
$N_{\mathrm{H}}\left(10^{22} \mathrm{~cm}^{-2}\right)$ & 5.9 & $3.0 \pm 0.4$ \\
Norm. factor & $\ldots$ & $3.2 \pm 2.3 \times 10^{-5}$ \\
$F_{X}^{\text {unabs }}\left(\mathrm{erg} \mathrm{s}^{-1} \mathrm{~cm}^{-2}\right)$ & $(1.7-9.2) \times 10^{-14}$ & $5.8 \pm 1.3 \times 10^{-14}$ \\
$\log \mathrm{L}_{X}\left(\mathrm{erg} \mathrm{s}^{-1}\right)$ & $(1.3-7.0) \times 10^{32}$ & $1.3 \times 10^{32}$ \\
$\log \left(\mathrm{L}_{\mathrm{X}} / \mathrm{L}_{\text {bol }}\right)$ & -7.2 to -6.4 & -7.2 \\
\hline
\end{tabular}

Notes. The following X-ray data were taken from the catalog of Muno et al. (2009): X-ray counts in the soft $\left(C_{\text {net }}^{\text {soft }}\right)$ and hard $\left(C_{\text {net }}^{\text {hard }}\right)$ energy bands, hardness ratios HR0 and HR2, photon flux $F_{\text {tot }}$, and average photon energy $\langle E\rangle$. All other values were derived here. For G359.973-0.008 (6), the values of $k T$, $N_{\mathrm{H}}$, and $F_{X}^{\text {unabs }}$ were derived from the infrared and X-ray photometry. For G359.925-0.049 (7), these same values were derived via a thermal plasma model fit, using the program XSPEC (Arnaud 1996), which also yielded the model normalization factor. Uncertainties are present when available.

In order to constrain the distance and nature of the X-ray sources, it is useful to examine the hardness ratio, defined as HR $=(h-s) /(h+s)$, where $h$ and $s$ are the fluxes in the hard and soft energy bands, respectively. The soft color, HR0, is defined by letting $h$ and $s$ be the fluxes in the respective $0.5-2.0 \mathrm{keV}$ and $2.0-3.3 \mathrm{keV}$ energy bands. The hard color, HR2, is defined by letting $h$ and $s$ be the fluxes in the $4.7-8.0 \mathrm{keV}$ and 3.3-4.7 keV energy bands, respectively. Galactic center sources, which typically lie behind a hydrogen absorption column of $N_{\mathrm{H}}>4 \times 10^{22} \mathrm{~cm}^{-2}$, will have most of their soft $\mathrm{X}$-ray photons absorbed by the interstellar medium (ISM), and will thus exhibit HR0 $\geqslant-0.175$ (Mauerhan et al. 2009a). Star 6 , with HR0 $=0.56$, definitely satisfies the criterion for Galactic center location, while star 7 , with $\mathrm{HR} 0=-0.17$ barely makes the cut. Compared with HR0, the more energetic photons used to calculate HR2 suffer significantly less absorption from intervening gas and dust, so they are more useful for constraining the intrinsic X-ray hardness. We compared the HR2 colors of these sources with those of the several dozen X-ray emitting massive stars in the GCR that were presented in Mauerhan et al. (2010b, their Figure 11). Star 6 is among the faintest X-ray sources that have a confirmed infrared counterpart. It was not significantly detected at energies above $4.7 \mathrm{keV}$, so its HR2 value is -1.0 . This is in contrast with most of several dozen known, massive stellar X-ray sources in the GCR, many of which have significant detections at energies up to $8.0 \mathrm{keV}$. This feature of the general population is presumed to be the result of emission from a relatively hard thermal plasma with $k T \gtrsim 2 \mathrm{keV}$. By contrast, the practically null detection of X-rays above $4.7 \mathrm{keV}$ energy from star 6 implies that it is a relatively faint and soft $\mathrm{X}$-ray source $(k T<1 \mathrm{keV}$, if it is thermal). Unfortunately, there are not enough counts to effectively model the X-ray spectrum. But if we assume a thermal plasma and guess a reasonable temperature, we can at least get an order-of-magnitude estimate 
Table 5

Distribution of WR Subtypes in the GCR

\begin{tabular}{lccccccccc}
\hline \hline \multicolumn{1}{c}{ Group } & WNE & WNL & WCE & WCL & WR $_{\text {tot }}$ & WC/WN & WCL/WC & WC/WR $_{\text {tot }}$ & References \\
\hline GCR “isolated" & 2 & 14 & 0 & 10 & 26 & 0.63 & 1.00 & 0.38 & $1,2,3,4,5,6$ \\
Arches & 0 & 15 & 0 & 0 & 15 & n/a & 0.00 & 0.00 & 7 \\
Quintuplet & 1 & 4 & 0 & 11 & 16 & 2.20 & 1.00 & 0.69 & $2,8,9$ \\
Central & 1 & 17 & 1 & 12 & 31 & 0.72 & 0.92 & 0.42 & 10 \\
GCR total & 4 & 50 & 1 & 33 & 88 & 0.63 & 0.97 & 0.39 & $\cdots$ \\
\hline
\end{tabular}

References. (1) This work; (2) Mauerhan et al. 2010b; (3) Cotera et al. 1999; (4) Homeier et al. 2003; (5) Muno et al. 2006; (6) Mikles et al. 2006; (7) Figer \& Kim 2002; (8) Liermann et al. 2009; (9) Figer et al. 1999c; (10) Paumard et al. 2006.

of the X-ray luminosity. To do this, we used the derived extinction values in Table 3 to calculate the corresponding hydrogen column density $\left(N_{\mathrm{H}}\right)$, using the $A_{V} / A_{K} \approx 16.1$ ratio from Nishiyama et al. (2008), assuming a negligible difference between $A_{K}$ and $A_{K_{\mathrm{s}}}$, and the $A_{V} / N_{\mathrm{H}}$ of Predehl \& Schmitt (1995), which gives $N_{\mathrm{H}} / A_{V}=1.8 \times 10^{21} \mathrm{~cm}^{-2} \mathrm{mag}^{-1}$. We obtained $N_{\mathrm{H}}=5.9 \times 10^{22} \mathrm{~cm}^{-2}$ for star 6 , which is consistent with the canonical average value of $N_{\mathrm{H}}=6 \times 10^{22} \mathrm{~cm}^{-2}$ that is typical for Galactic center $\mathrm{X}$-ray sources. Using the observed $0.5-8.0 \mathrm{keV}$ energy flux of $F_{X}=7.3 \times 10^{-16} \mathrm{erg} \mathrm{s}^{-1} \mathrm{~cm}^{-2}$ for this source, we employed the PIMMS ${ }^{7}$ simulator to calculate the absorption-corrected X-ray flux. We assumed an intrinsic thermal plasma energy value range of $k T=0.6-1 \mathrm{keV}$, which is appropriate; after all, the faintness of this source and its lack of high energy emission (low HR2 value), compared with the other GCR X-ray sources of Mauerhan et al. (2010b), imply that the plasma temperature is unlikely to be any hotter than this. PIMMS predicts an unabsorbed X-ray flux in the range of (1.7-9.2) $\times 10^{-14} \mathrm{erg} \mathrm{s}^{-1} \mathrm{~cm}^{-2}$ for star 6. Accounting for the distance of $8 \mathrm{kpc}$, this implies an X-ray luminosity in the range of (1.3-7.0) $\times 10^{32} \mathrm{erg} \mathrm{s}^{-1}$ in the $0.5-8.0 \mathrm{keV}$ energy band. Thus, assuming $k T$ is between 0.6 and $1 \mathrm{keV}$, the $\mathrm{X}$-ray to bolometric luminosity ratio of star 6 is in the range of $\log \left(\mathrm{L}_{\mathrm{X}} / \mathrm{L}_{\mathrm{bol}}\right)=-7.2$ to -6.4

The other O4-6I star, 7, has a relatively large X-ray flux in the soft energy range $(0.5-2.0 \mathrm{keV})$ compared with star 6 and the other massive stellar X-ray sources in the GCR. It is also relatively bright overall. This is not surprising, since both the photometry and the extinction value that we calculated for the stellar counterpart in Table 3 imply a foreground distance of $3.6 \mathrm{kpc}$. So, if the X-ray-detected O4-6I stars 6 and 7 have the same intrinsic nature, then we should expect the foreground star 7 to appear brighter. Fortunately, star 7 has enough counts to warrant a fitting of its X-ray spectrum, which can better constrain the physical parameters of the X-ray emission. To do this, we used the program XSPEC (Arnaud 1996). First, the $\mathrm{X}$-ray spectral data were binned to have at least 30 counts in each energy bin. Then, we performed least squares fits to the data by trying out two different emission models, including a power-law model and a thermal plasma model. The power-law fit required an unrealistically steep photon index of $\Gamma \approx 5$, so we do not regard this as a viable model for star 7 . However, the thermal plasma model fit the data reasonably well. The parameters fit by the model were $N_{\mathrm{H}}, k T$, and a normalization factor that is proportional to the emission measure. For the iterative fitting procedure, we used initial guess values of $N_{\mathrm{H}}=3.4 \times 10^{22} \mathrm{~cm}^{-2}$, derived from the infrared photometry, and $k T=1 \mathrm{keV}$. After 30 fitting iterations, XSPEC returned values of $N_{\mathrm{H}}=(3.0 \pm 0.4) \times 10^{22} \mathrm{~cm}^{-2}, k T=0.9 \pm 0.2 \mathrm{keV}$,

\footnotetext{
7 http://heasarc.gsfc.nasa.gov/Tools/w3pimms.html
}

and an energy flux of $F_{X}=4.2 \times 10^{-15} \mathrm{erg} \mathrm{s}^{-1} \mathrm{~cm}^{-2}$. The reduced $\chi^{2}$ of the fit was 0.7 for 13 degrees of freedom. Using these parameters we calculated an absorbtion-corrected X-ray flux of $F_{X}^{\text {unabs }}=(5.8 \pm 1.3) \times 10^{-14} \mathrm{erg} \mathrm{s}^{-1} \mathrm{~cm}^{-2}$. Using the derived distance of $3.6 \mathrm{kpc}$, we calculated an X-ray luminosity of $L_{X}=1.3 \times 10^{32} \mathrm{erg} \mathrm{s}^{-1}$ for star 7, which implies an X-ray to bolometric luminosity ratio of $\log \left(\mathrm{L}_{\mathrm{X}} / \mathrm{L}_{\mathrm{bol}}\right)=-7.2$.

The estimated plasma temperatures and X-ray luminosities of stars 6 and 7 are consistent with the values that have been measured for the GCR O4-6I stars in the sample of Mauerhan et al. (2010b), and the O4-6I stars in the Cygnus OB2 sample of Albacete Colombo et al. (2007). O stars typically exhibit $\log \left(\mathrm{L}_{\mathrm{X}} / \mathrm{L}_{\mathrm{bol}}\right) \approx-7$, and this is true for both single stars and those in massive binaries (Oskinova 2005). This relation is a result of the connection between the strength of radiatively driven winds and the temperature of the resulting shocks, which may occur inside the stellar wind via turbulence-induced micro shocks, or from wind collision if the $\mathrm{O}$ star is in a massive binary. However, the plasma temperatures reached via the interwind micro-shock process typically have $k T<1 \mathrm{keV}$, while shocks in colliding-wind binaries generate higher temperatures $(k T \gtrsim 1-2 \mathrm{keV})$ and produce harder $\mathrm{X}$-ray spectra. Our derived value of $k T=0.9 \pm 0.2 \mathrm{keV}$ is insufficient to effectively discriminate between these two mechanisms. Although the average photon energy of both sources is $\langle E\rangle=2.5-3.0 \mathrm{keV}$, these photons could simply be the tail of a much softer intrinsic spectrum whose lower energy photons were absorbed by the ISM. Alternatively, our assumption of a single temperature plasma for star 7 might be too simplistic, and the highest energy photons detected from this source could actually be from an intrinsic hard component with $k T \gtrsim 1-2 \mathrm{keV}$. But without more counts in the softer energy bands, we cannot reliably fit a realistic two-temperature or three-temperature plasma model to the data, which is generally the case for high X-ray flux $\mathrm{O}$ stars in Cygnus. In conclusion, the X-ray properties of stars 6 and 7 are consistent with thermal emission from either single $\mathrm{O}$ stars or those in colliding-wind binaries, although we currently have no good reason to invoke binarity for either source.

\section{DISCUSSION}

\subsection{How Complete is the WR Sample in the Central $\approx 90$ pc of the Galaxy?}

There are currently 88 identified WRs in the GCR, including all known WNE (WN4-6), WNL (WN7-9), WCE (WC4-7), and WCL (WC8-9) stars identified in the field (including those identified outside of the $\mathrm{P} \alpha$ survey area), and those identified in the Arches, Quintuplet, and Central clusters. Their subtype statistics are presented in Table 5. The most noteworthy characteristic of the WR subtype distribution is the high latetype to early-type ratio for both WC and WN stars; out of the 


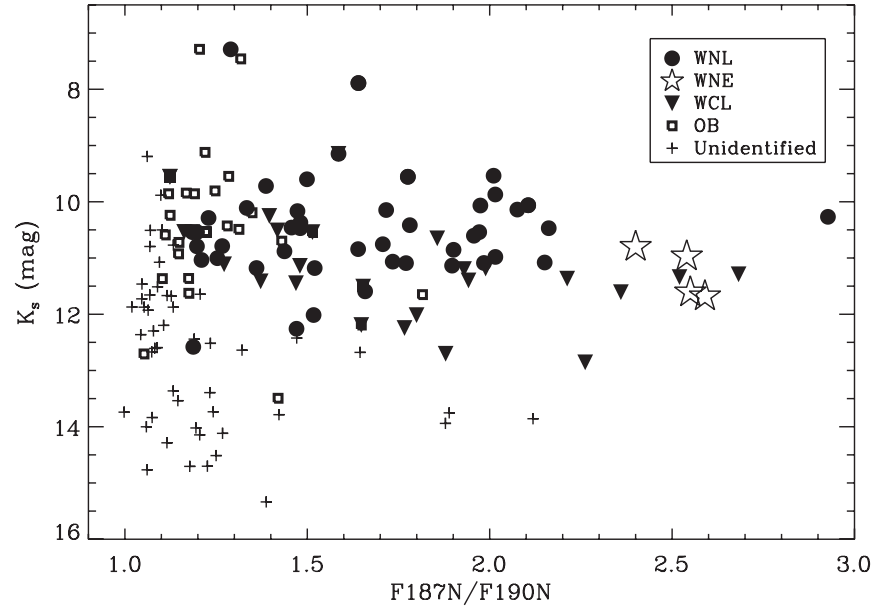

Figure 7. Plot of observed line strength $(\mathrm{F} 187 \mathrm{~N} / \mathrm{F} 190 \mathrm{~N})$ vs. $K_{s}$-band Sirius magnitude for unidentified candidate $\mathrm{P} \alpha$-excess sources (crosses), and for confirmed massive stars. The brightest known sources $\left(K_{s} \lesssim 8 \mathrm{mag}\right)$, which are saturated in the Sirius catalog, were plotted using their 2 MASS photometry. The figure includes objects from the Arches, Quintuplet, and Central Parsec clusters, and from the GCR field. Stars are marked according to their approximate spectral type, defined in the legend at the upper right of the figure. The brightness distribution of unidentified sources suggests that the sample of WN types in the survey might be near completion.

54 WN stars, only 4 are WNE types; and out of 34 WC stars, only 1 is a WCE type. WC stars comprise $38 \%$ of the total WR population (47\% if we exclude the Arches cluster, which is too young to have produced WC stars).

Figure 7 shows a plot of $\mathrm{P} \alpha$ line strength (defined here as the F187N/F190N flux ratio) versus $K_{s}$-band magnitude for known WRs in the GCR and the remaining unidentified P $\alpha$-excess candidates in the survey area (Wang et al. 2010; H. Dong et al. 2011, in preparation). The known sources in Figure 7 include emission-line stars from the GCR field, and from the Arches, Quintuplet, and Central Parsec clusters. The $K_{s}$-band values of unidentified sources were obtained by cross-correlating the positions of the P $\alpha$-excess candidates with sources from the Sirius and 2MASS catalogs (we make a note of caution that a few of the unidentified $\mathrm{P} \alpha$ sources having the faintest $K_{s}$ matches might be spurious associations in this crowded region, and that Sirius magnitudes may have significant systematic errors because of source confusion, especially near the Central Parsec cluster). The data in Figure 7 suggest that all but a few of the remaining unidentified $\mathrm{P} \alpha$ candidates occupy a brightness range of $K_{s}$ and F187N/F190N that is systematically offset from the range occupied by confirmed WRs. Thus, an important question naturally arises as to the completeness of the WR sample at the inner $(l, b) \approx 90 \times 35 \mathrm{pc}^{2}$ around Sgr A* (the survey area).

WNL stars, which almost completely dominate the current WN sample, have F187N/F190N ratios between 1.2 and 2.9, and have bright counterparts with $K_{s} \approx 7.5-12$ mag. WNL types are expected to generally have $K \lesssim 12$ mag when observed in the GCR (e.g., see Figer 1995, their Figure 25). Thus, we suspect that only a few WNL stars, at most, could remain unidentified in the survey area. This also appears to be the case for WNE types; those confirmed thus far, all of which have subtypes in the WN5-6 range, have several of the highest F187N/ F190N ratios in the entire sample of WRs. None of the unidentified $\mathrm{P} \alpha$-excess candidates have $\mathrm{F} 187 \mathrm{~N} / \mathrm{F} 190 \mathrm{~N}$ ratios this large. Earlier WNE stars of subtype WN3-4 have not yet been found in the GCR. Although they are intrinsically the faintest of the WN stars $\left(M_{K} \approx-3.1 \mathrm{mag}\right.$; Crowther et al. 2006), having expected
$K$-band magnitudes as faint as $\approx 14$ in the GCR, WN3-4 stars will exhibit $\mathrm{P} \alpha$ excess similar to the WN5-6 stars. Therefore, they should still be easily detectable as relatively strong sources of $\mathrm{P} \alpha$ excess, ${ }^{8}$ modulo the presence of a bright, diffuse background. Thus, although Figure 7 indicates that several WN stars could remain unidentified, we suspect the WN sample in the survey area is near completion nonetheless. This implies that WNL types heavily dominate the GCR WN population, and that WNE subtypes are intrinsically rare in the region.

The situation is less clear for WC stars. Their F187N/F190N ratios span a range of values similar to the distribution of WNL types, but the faint end of their expected brightness distribution in the GCR extends down to $K \approx 15$ mag for the earliest WCE subtypes (Figer 1995). This has been confirmed by photometry of the WC5-6 star IRS 3E of the Central Parsec cluster (E58 from Paumard et al. 2006), which has $K=15.0$ mag and is the only WCE star identified in the GCR to date. Unfortunately, the F187N/F190N ratio for this source could not be measured because of source confusion and the strong, diffuse background emission from Sgr A West. Even with relatively large intrinsic F187N/F190N ratios, however, sources as faint as IRS 3E will have large photometric uncertainties that could render them weak or undetectable sources of $\mathrm{P} \alpha$ excess in the HST/NICMOS survey (H. Dong et al. 2011, in preparation). Thus, it is difficult to ascertain the detectability of a hypothetical population of WCE types in the GCR, and we cannot reliably estimate the potential contribution of such stars to the sample of unidentified sources in Figure 7. The same is true for WCL types, which can also be very faint ( $K \lesssim 14 \mathrm{mag}$ ) and whose line strengths can be heavily diluted in cases where there is significant flux contribution from a host dust continuum. Extremely dusty WCL stars (DWCL; Williams et al. 1987), like the Quintuplet proper members, whose emission lines have been completely diluted by a thermal continuum or obscuring dust screen, are not detectable $\mathrm{P} \alpha$ excess sources at all; so, some of these stellar types could remain unidentified. However, DWCL stars are very bright infrared sources ( $K_{s} \approx 6-7 \mathrm{mag}$ ) and typically have $\mathrm{X}$-ray counterparts. Based on these properties, the statistics of X-ray/infrared matches from Mauerhan et al. (2009a), combined with the spectroscopic survey results of Mauerhan et al. (2010b), imply that most, if not all, DWCL stars in the survey area should have been found already. Still, less extreme WC9d stars with diluted emission lines that are not detectable X-ray sources might remain elusive.

The near completeness of the WN stars in the survey area implies that apparent dominance of WNL stars over WNE stars is an intrinsic property of the GCR. Although the sample of WC stars is probably not complete, we also suspect that WCL stars are intrinsically dominant over WCE types. The reasons for this are as follows. It has been well established that WR subtype distributions are correlated with environmental metallicity; this has been established for the Milky Way and other galaxies of the Local Group (Massey 1996). WNE and WCE stars appear to be rare in relatively high-metallicity environments $\left(Z \geqslant Z_{\odot}\right)$, such as the inner Galaxy, but are common in lowmetallicity environments, such as the outer Galaxy $\left(Z \geqslant Z_{\odot}\right)$, and the Large Magellanic Cloud $\left(Z \ll Z_{\odot}\right)$. This observed trend has been interpreted as a result of the metallicity dependence

\footnotetext{
8 We have confirmed this statement by examining the $H K$ spectra of WN3-6 and WC4-6 stars obtained from the ground (not shown here). The spectra of the WNE stars exhibit large flux at $\lambda 1.87 \mu \mathrm{m}$ that can penetrate the severe atmospheric absorption at this wavelength, which implies that such stars will generally have high $\mathrm{F} 187 \mathrm{~N} / \mathrm{F} 190 \mathrm{~N}$ ratios.
} 


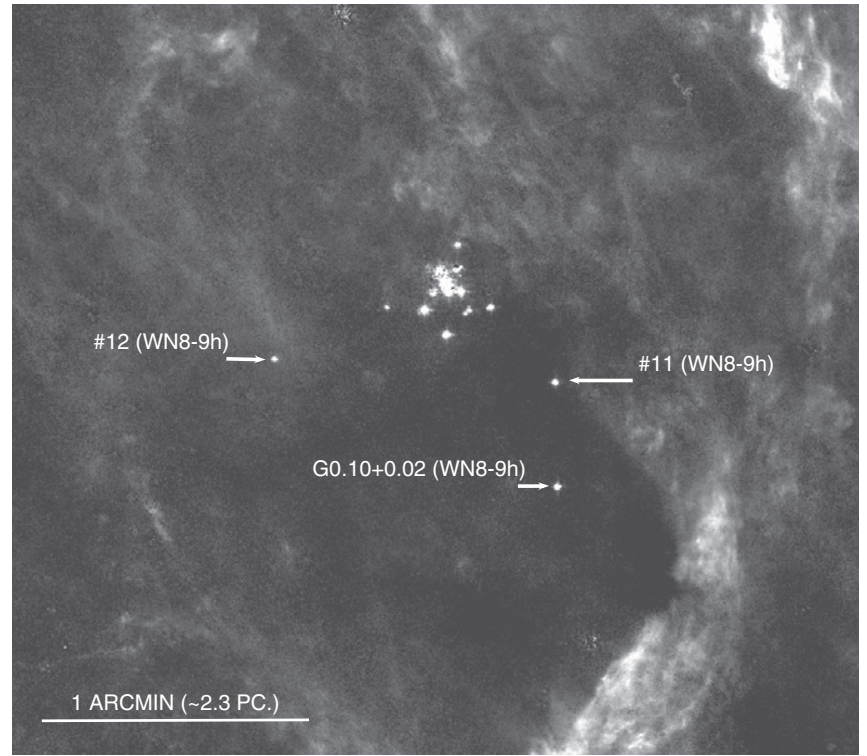

Figure 8. HST/NICMOS $\mathrm{P} \alpha$ survey image from Wang et al. (2010) of the Arches cluster region and nearby WNh stars. The marked stars 11 and 12 are new discoveries with the exception of G0.10+0.02, which was reported in Cotera et al. (1999). The surrounding stars all lie within 1-2 pc, in projection, of the cluster (beyond the tidal radius of $\approx 1 \mathrm{pc}$ ), and they may have escaped from it. North is up and east is to the left in this image.

of WR winds (see Crowther 2007 and references therein for the following discussion). For WN stars, the higher wind density that results from increased metallicity leads to more efficient atomic recombination from high-ionization stages (e.g., $\mathrm{N}^{5+}$ ) to lower ions (e.g., $\mathrm{N}^{3+}$ ), which effectively increases the trend toward later spectral types. Alternatively, decreased recombination efficiency and reduced nitrogen content at lower metallicity result in a trend toward earlier WN subtypes. For WC stars in high-metallicity environments, higher wind densities lead to an increased emission strength of C III with respect to C IV. Since WCL and WCE stars are classified as such based upon the relative strengths of $\mathrm{C}$ III and $\mathrm{C}$ IV emission lines, WC stars in environments with $Z \geqslant Z_{\odot}$ will tend to manifest as WCL subtypes (C III stronger than $C_{\text {IV }}$ ). Although this effect was originally suggested by Crowther et al. (2002) to be an important factor for the optical classification of WC stars (specifically for $\mathrm{C}$ III at $\lambda 5696 \AA$ ), the effect of elevated $\mathrm{C}$ III relative to $\mathrm{C}$ IV for increasing wind density is likely to be mirrored in the infrared spectrum of WC stars as well. Thus, the dominance of WNL and WCL stars over WNE and WCE stars in the GCR is a natural consequence of the fact that the metallicity of the region is at least solar (e.g., see Davies et al. 2009 and references therein), if not supersolar (Cunha et al. 2007; Najarro et al. 2009).

\subsection{Rogue Massive Stars from the Arches and Quintuplet Clusters}

Figures 8 and 9 show the distribution of "isolated" massive stars very near the Arches and Quintuplet clusters. For the Arches, there are three WNh stars that lie within $\approx 2 \mathrm{pc}$, in projection, of the cluster. The magnitudes and spectral types of these outer WNh stars are very similar to the brightest Arches members, some of which have estimated initial masses above $100 M_{\odot}$ (Figer \& Kim 2002). Based on the spectral similarities and the close spatial proximity, we suggest that the three massive stars surrounding the Arches cluster are coeval with the system. The main uncertainty is with regard

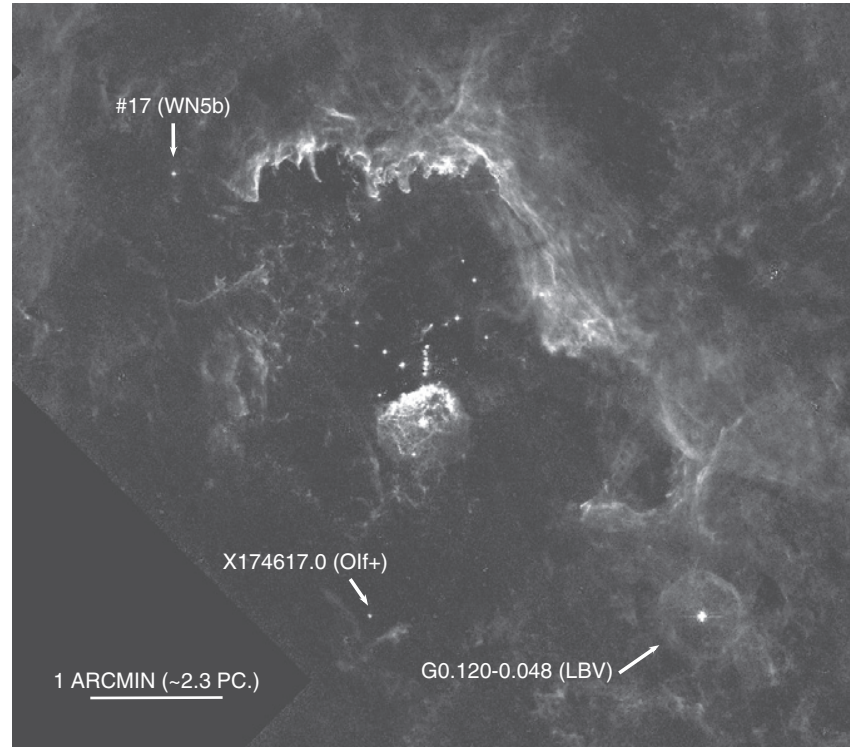

Figure 9. HST/NICMOS $\mathrm{P} \alpha$ survey image of the Sickle region from Wang et al. (2010), which contains the Quintuplet cluster and nearby isolated massive stars, including the $\mathrm{O6If}^{+}$X-ray source X174517.0 from Mauerhan et al. (2007), the LBV G0.120-0.048 from Mauerhan et al. (2010b), and the new WN5b star 17 of this work. All of the unmarked point sources are known massive stars spectroscopically identified in Figer et al. (1999c). North is up and east is to the left in this image.

to their current kinematical state. One might expect that mass segregation via dynamical friction should concentrate massive stars like these toward the cluster center. However, higher order processes occurring at the cluster center might result in an alternative effect. Indeed, the Arches cluster is the most dense system known in the Galaxy $\left(3 \times 10^{5} M_{\odot} \mathrm{pc}^{-3}\right.$; Figer et al. 1999a). As such, the central region of this cluster must be the site of frequent gravitational encounters between massive stars and binaries, including binary captures, disruptions, and exchanges. Through such interactions, one might expect a few massive stars to be gravitationally "kicked" from time to time beyond the tidal radius, which is $\approx 1 \mathrm{pc}$ for the Arches and Quintuplet (Portegies Zwart et al. 2002). This could be the case for stars 11 and 12 of this work, and G0.10+0.02 from Cotera et al. (1999), all of which lie well beyond the Arches cluster's tidal radius. Given that the internal velocity dispersion of the Arches cluster is $\sim 10 \mathrm{~km} \mathrm{~s}^{-1}$ (Figer \& Kim 2002), a massive star would not require an unusually large gravitational "kick" to escape the cluster. Alternatively, one or more of these stars could have formed at the same time and from the same cloud as the main cluster but never became bound to the system. If this is the case then the stars could simply be following a path that is in tandem with that of the cluster, without orbiting the cluster center. These possibilities may also be applicable to the massive stars surrounding the Quintuplet cluster. Mauerhan et al. (2007) suggested that the O6If ${ }^{+}$X-ray source CXOGC J174617.0-285131, a suspected colliding-wind binary, may have originated in the Quintuplet, while the same might be true for the nearby LBV G0.120-0.048 (Mauerhan et al. 2010a).

Observationally distinguishing between the various possible origins of the stars surrounding the Arches and Quintuplet clusters would be very difficult using radial velocities alone, since both ejected stars and comoving stars that were never bound to the clusters would exhibit similar radial velocities, perhaps differing on the order of only $10 \mathrm{~km} \mathrm{~s}^{-1}$. Proper 


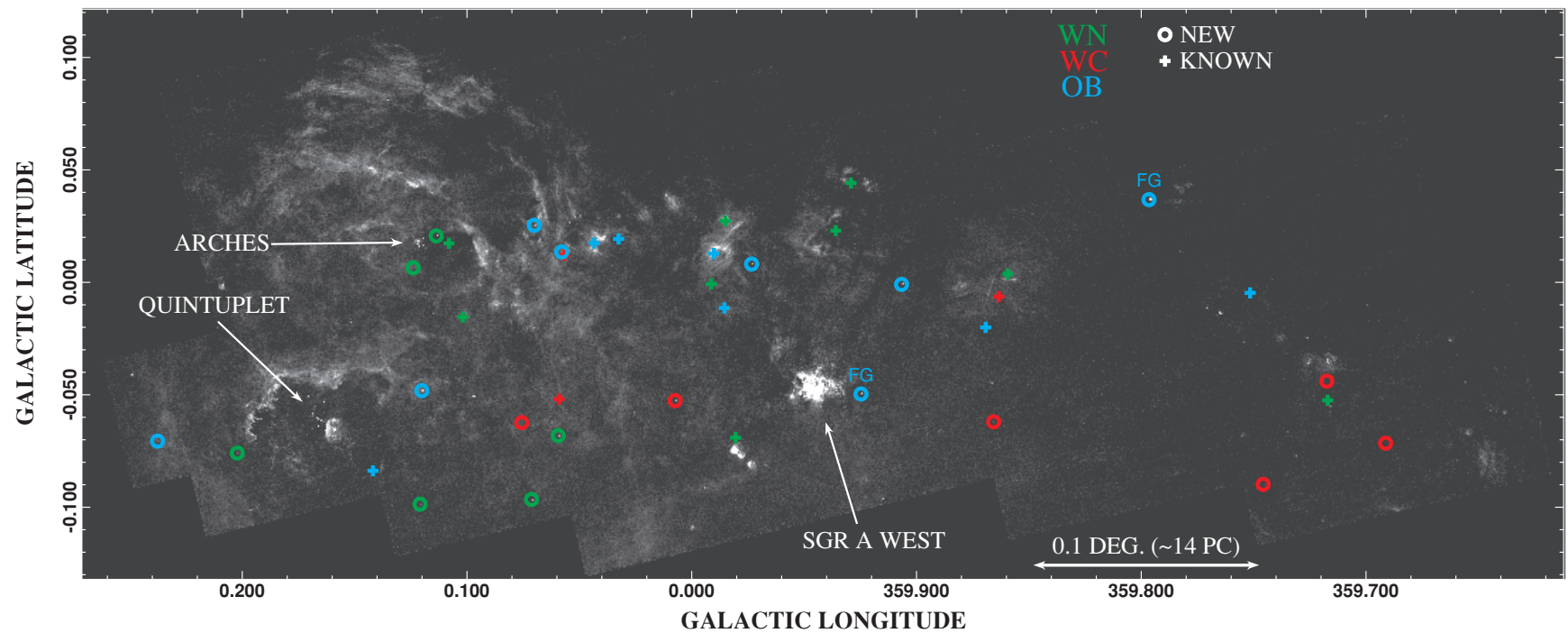

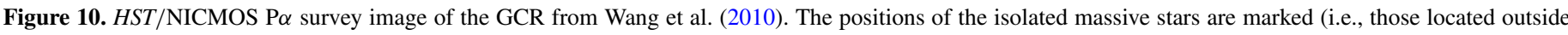

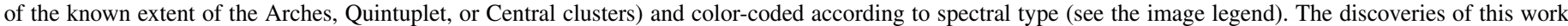
(circles) are marked separately from the known sources (crosses). The two foreground O stars are labeled "FG." Bona fide cluster members are not marked.

motion measurements, however, would allow for the derivation of a three-dimensional space velocity vector, which would better elucidate the association between these stars and the clusters, and could provide evidence for ejection, specifically. Such measurements are within the capabilities of $10 \mathrm{~m}$ class telescopes utilizing adaptive optics. For example, Stolte et al. (2008) used proper motion measurements over a 4.3 year baseline to derive a three-dimensional space velocity of $232 \pm$ $30 \mathrm{~km} \mathrm{~s}^{-1}$ for the Arches cluster. As coeval siblings of the Arches cluster stars, we might expect stars 11,12 , and G0.10+0.02 to have similar velocity, whether they were ejected from the cluster or are just comoving with it. Multiple epoch measurements with longer time baselines, however, could discriminate between ejection and comoving scenarios by achieving the required accuracy to obtain a velocity difference vector between the individual star vectors and the average cluster vector. If the stars were ejected from the cluster, we would expect these difference vectors to point away from the cluster.

\subsection{Are there Unidentified Stellar Clusters in the GCR?}

The continuum-subtracted $\mathrm{P} \alpha$ mosaic of the inner $(l, b) \approx$ $90 \times 35 \mathrm{pc}^{2}$ of the GCR from Wang et al. (2010) is shown in Figure 10, where the positions of the known clusters and the "isolated" massive stars are illustrated. Aside from the several stars potentially associated with the Arches and Quintuplet clusters, some of the other new discoveries appear to reside in regions that were heretofore unidentified as potential associations. There is a noteworthy association of three WC9 stars, a WN7, and an OB supergiant at $(l, b) \approx(359.68-359.76,-0.05)$. Several promising unidentified candidates having $K_{s} \approx 12.4$ and $\approx 12.7 \mathrm{mag}$ and $\mathrm{F} 187 \mathrm{~N} / \mathrm{F} 190 \mathrm{~N}$ ratios consistent with them being WC or OB stars also lie in this region. This area is far from any of the known clusters, so, these stars might be the brightest representative members of a bona fide association. There is a more highly concentrated group containing a WC9d, an Ofpe/ WN9, and a B2 Ia star at $(l, b) \approx(359.86,0.00)$, originally identified as a potentially coeval system by Mauerhan et al. (2007). In addition, there appears to be a noteworthy collection of two WC9 stars and an O supergiant near $(l, b) \approx(0.07$,
-0.06), and a significant concentration of WN stars near $(l, b) \approx(359.98,0.00)$ and $(l, b) \approx(0.05,0.02)$, including the central stars of the radio $\mathrm{H}$ II regions $\mathrm{H} 1, \mathrm{H} 2, \mathrm{H} 5$, and $\mathrm{H} 8$ (Zhao et al. 1993).

Could these associations of massive stars represent the "tip of the iceberg" of stellar clusters that are less compact than the Arches and Quintuplet? There is no measured stellar density enhancement in the vicinity of isolated massive stars in the GCR (Wang et al. 2010; H. Dong et al. 2011, in preparation). But because of the strong tidal field in the GCR, stellar clusters like the Arches and Quintuplet will dissolve on a timescale of $\approx 10 \mathrm{Myr}$ (Kim et al. 1999), while the time it takes for less extreme stellar clusters to fade into the background may be shorter than this. According to Portegies Zwart et al. (2002), the cluster dissolution timescale is a sensitive function of galactocentric radius, initial cluster mass, and initial density, such that less compact stellar clusters could become invisible as stellar density enhancements on a shorter timescale than the Arches or Quintuplet ages, or could even persist as undetectable entities for their entire lives. In such cases, an Earth-based observer undertaking a narrowband imaging survey of the GCR might see a small group of evolved emission-line stars with no obvious surrounding stellar cluster. This could be the case for the associations that we have noted above.

As an alternative explanation for the "isolated" massive stars, there may be two modes of massive star formation operating in the GCR, one that produces dense starburst systems like the Arches and Quintuplet, and one that produces massive stars in small, sparse associations or in total isolation. Currently, we cannot discriminate between cluster and isolated star formation origins; but continued surveys for coeval massive stars in the vicinity of the new emission-line stars could provide inroads to solving this problem.

The project is partly supported by NASA through a grant HST-GO-11120 from the Space Telescope Science Institute, which is operated by the Association of Universities for Research in Astronomy, Inc., under NASA contract NAS 5-26555. 


\section{REFERENCES}

Albacete Colombo, J. F., Flaccomio, E., Micela, G., Sciortino, S., \& Damiani, F. 2007, A\&A, 464, 211

Arnaud, K. A. 1996, in ASP Conf. Ser. 101, Astronomical Data Analysis Software and Systems, ed. G. H. Jacoby \& J. Barnes (San Francisco, CA: ASP), 17

Churchwell, E., et al. 2009, PASP, 121, 213

Cotera, A. S., Simpson, J. P., Erickson, E. F., Colgan, S. W. J., Burton, M. G., \& Allen, D. A. 1999, ApJ, 510, 747

Crowther, P. A. 2007, ARA\&A, 45, 177

Crowther, P. A., Dessart, L., Hillier, D. J., Abbott, J. B., \& Fullerton, A. W. 2002, A\&A, 392, 653

Crowther, P. A., Hadfield, L. J., Clark, J. S., Negueruela, I., \& Vacca, W. D. 2006, MNRAS, 372, 1407

Cunha, K., Sellgren, K., Smith, V. V., Ramirez, S. V., Blum, R. D., \& Terndrup, D. M. 2007, ApJ, 669, 1011

Cushing, M. C., Vacca, W. D., \& Rayner, J. T. 2004, PASP, 116, 362

Cutri, R. M., et al. 2003, 2MASS All Sky Catalog of Point Sources (The IRSA 2MASS All-Sky Point Source Catalog, NASA/IPAC Infrared Science Archive), http://irsa.ipac.caltech.edu/applications/Gator/

Davies, B., Origlia, L., Kudritzki, R.-P., Figer, D. F., Rich, R. M., \& Najarro, F. 2009, ApJ, 694, 46

Depoy, D. L., Atwood, B., Byard, P. L., Frogel, J., \& O’Brien, T. P. 1993, Proc. SPIE, 1946, 667

Figer, D. F. 1995, PhD thesis, Univ. California, Los Angeles

Figer, D. F., \& Kim, S. S. 2002, in ASP Conf. Ser. 263, Stellar Collisions, Mergers, and their Consequences, ed. M. Shara (San Francisco, CA: ASP), 287

Figer, D. F., Kim, S. S., Morris, M., Serabyn, E., Rich, R. M., \& McLean, I. S. 1999a, ApJ, 525, 750

Figer, D. F., McLean, I. S., \& Morris, M. 1999b, ApJ, 514, 202

Figer, D. F., McLean, I. S., \& Najarro, F. 1997, ApJ, 486, 420

Figer, D. F., Morris, M., Geballe, T. R., Rich, R. M., Serabyn, E., McLean, I. S., Puetter, R. C., \& Yahil, A. 1999c, ApJ, 525, 759

Figer, D. F., Najarro, F., Morris, M., McLean, I. S., Geballe, T. R., Ghez, A. M., \& Langer, N. 1998, ApJ, 506, 384

Figer, D. F., Rich, R. M., Kim, S. S., Morris, M., \& Serabyn, E. 2004, ApJ, 601, 319

Geballe, T. R., Najarro, F., \& Figer, D. F. 2000, ApJ, 530, L97

Ghez, A. M., et al. 2003, ApJ, 586, L127

Glass, I. S., Moneti, A., \& Moorwood, A. F. M. 1990, MNRAS, 242, 55P

Hadfield, L. J., van Dyk, S. D., Morris, P. W., Smith, J. D., Marston, A. P., \& Peterson, D. E. 2007, MNRAS, 376, 248

Hanson, M. M., Conti, P. S., \& Rieke, M. J. 1996, ApJS, 107, 281

Homeier, N. L., Blum, R. D., Pasquali, A., Conti, P. S., \& Damineli, A. 2003, A\&A, 408, 153

Kim, S. S., Morris, M., \& Lee, H. M. 1999, ApJ, 525, 228

Krabbe, A., Genzel, R., Drapatz, S., \& Rotaciuc, V. 1991, ApJ, 382, L19

Liermann, A., Hamann, W.-R., \& Oskinova, L. M. 2009, A\&A, 494, 1137

Martins, F., Hillier, D. J., Paumard, T., Eisenhauer, F., Ott, T., \& Genzel, R. 2008, A\&A, 478, 219
Martins, F., \& Plez, B. 2006, A\&A, 457, 637

Massey, P. 1996, in Liege Int. Astrophys. Colloq. 33, Wolf-Rayet Stars in the Framework of Stellar Evolution, ed. J. M. Vreux, A. Detal, D. Fraipont-Caro, E. Gosset, \& G. Rauw (Liége: Univ. Liége, Institut d'Astrophysique), 361

Mauerhan, J. C., Morris, M. R., Cotera, A., Dong, H., Wang, Q. D., Stolovy, S. R., Lang, C., \& Glass, I. S. 2010a, ApJ, 713, L33

Mauerhan, J. C., Muno, M. P., \& Morris, M. 2007, ApJ, 662, 574 (MMM07)

Mauerhan, J. C., Muno, M. P., Morris, M. R., Bauer, F. E., Nishiyama, S., \& Nagata, T. 2009a, ApJ, 703, 30

Mauerhan, J. C., Muno, M. P., Morris, M. R., Stolovy, S. R., \& Cotera, A. 2010b, ApJ, 710, 706

Mauerhan, J. C., van Dyk, S. D., \& Morris, P. W. 2009b, PASP, 121, 591

Mikles, V. J., Eikenberry, S. S., Muno, M. P., Bandyopadhyay, R. M., \& Patel, S. 2006, ApJ, 651, 408

Morris, M., \& Serabyn, E. 1996, ARA\&A, 34, 645

Morris, P. W., Eenens, P. R. J., Hanson, M. M., Conti, P. S., \& Blum, R. D 1996, ApJ, 470, 597

Muno, M. P., Bower, G. C., Burgasser, A. J., Baganoff, F. K., Morris, M. R., \& Brandt, W. N. 2006, ApJ, 638, 183

Muno, M. P., et al. 2009, ApJS, 181, 110

Nagata, T., Woodward, C. E., Shure, M., \& Kobayashi, N. 1995, AJ, 109, 1676

Nagata, T., Woodward, C. E., Shure, M., Pipher, J. L., \& Okuda, H. 1990, ApJ, 351,83

Najarro, F., Figer, D. F., Hillier, D. J., Geballe, T. R., \& Kudritzki, R. P. 2009, ApJ, 691, 1816

Nishiyama, S., Nagata, T., Tamura, M., Kandori, R., Hatano, H., Sato, S., \& Sugitani, K. 2008, ApJ, 680, 1174

Nishiyama, S., et al. 2006, ApJ, 638, 839

Okuda, H., et al. 1990, ApJ, 351, 89

Oskinova, L. M. 2005, MNRAS, 361, 679

Paumard, T., et al. 2006, ApJ, 643, 1011

Portegies Zwart, S. F., Makino, J., McMillan, S. L. W., \& Hut, P. 2002, ApJ, 565,265

Predehl, P., \& Schmitt, J. H. M. M. 1995, A\&A, 293, 889

Ramsay Howatt, S. K., et al. 2004, Proc. SPIE, 5492, 1160

Rayner, J. T., Toomey, D. W., Onaka, P. M., Denault, A. J., Stahlberger, W. E., Vacca, W. D., Cushing, M. C., \& Wang, S. 2003, PASP, 115, 362

Reid, M. J. 1993, ARA\&A, 31, 345

Schödel, R., et al. 2002, Nature, 419, 694

Stolte, A., Ghez, A. M., Morris, M., Lu, J. R., Brandner, W., \& Matthews, K 2008, ApJ, 675, 1278

Tinney, C. G., et al. 2004, Proc. SPIE, 5492, 998

Vacca, W. D., Cushing, M. C., \& Rayner, J. T. 2003, PASP, 115, 389

Walborn, N. R. 1971, ApJS, 23, 257

Wang, Q. D., et al. 2010, MNRAS, 402, 895

Williams, P. M., van der Hucht, K. A., \& Rauw, G. 2005, in Proc. JENAM, Massive Stars and High-Energy Emission in OB Associations, ed. G. Rauw, Y. Nazé, R. Blomme, \& E. Gosset (Liége: Univ. Liége), 65

Williams, P. M., van der Hucht, K. A., \& The, P. S. 1987, A\&A, 182, 91

Zhao, J.-H., Desai, K., Goss, W. M., \& Yusef-Zadeh, F. 1993, ApJ, 418, 235 